\title{
Striking antibody evasion manifested by the Omicron variant of SARS-CoV-2
}

https://doi.org/10.1038/s41586-021-04388-0

Received: 14 December 2021

Accepted: 23 December 2021

Published online: 23 December 2021

Check for updates

\author{
Lihong Liu ${ }^{1,7}$, Sho Iketani ${ }^{1,2,7}$, Yicheng Guo ${ }^{1,7}$, Jasper F.-W. Chan ${ }^{3,4,7}$, Maple Wang ${ }^{1,7}$, Liyuan Liu ${ }^{5,7}$, \\ Yang Luo', Hin $\mathrm{Chu}^{3,4}$, Yiming Huang ${ }^{5}$, Manoj S. Nair', Jian Yu', Kenn K.-H. Chik ${ }^{4}$, \\ Terrence T.-T. Yuen ${ }^{3}$, Chaemin Yoon ${ }^{3}$, Kelvin K.-W. To ${ }^{3,4}$, Honglin Chen ${ }^{3,4}$, Michael T. Yin ${ }^{1,6}$, \\ Magdalena E. Sobieszczyk ${ }^{1,6}$, Yaoxing Huang', Harris H. Wang ${ }^{5}$, Zizhang Sheng', \\ Kwok-Yung Yuen ${ }^{3,4} \&$ David D. Ho
}

\begin{abstract}
The B.1.1.529/Omicron variant of SARS-CoV-2 was only recently detected in southern Africa, but its subsequent spread has been extensive, both regionally and globally'. It is expected to become dominant in the coming weeks ${ }^{2}$, probably due to enhanced transmissibility. A striking feature of this variant is the large number of spike mutations ${ }^{3}$ that pose a threat to the efficacy of current COVID-19 vaccines and antibody therapies ${ }^{4}$. This concern is amplified by the findings of our study. Here we found that B.1.1.529 is markedly resistant to neutralization by serum not only from patients who recovered from COVID-19, but also from individuals who were vaccinated with one of the four widely used COVID-19 vaccines. Even serum from individuals who were vaccinated and received a booster dose of mRNA-based vaccines exhibited substantially diminished neutralizing activity against B.1.1.529. By evaluating a panel of monoclonal antibodies against all known epitope clusters on the spike protein, we noted that the activity of 17 out of the 19 antibodies tested were either abolished or impaired, including ones that are currently authorized or approved for use in patients. Moreover, we also identified four new spike mutations (S371L, N440K, G446S and Q493R) that confer greater antibody resistance on B.1.1.529. The Omicron variant presents a serious threat to many existing COVID-19 vaccines and therapies, compelling the development of new interventions that anticipate the evolutionary trajectory of SARS-CoV-2.
\end{abstract}

The COVID-19 pandemic continues as the causative agent SARS-CoV-2 continues to evolve. Many diverse viral variants have emerged (Fig. 1a), each characterized by mutations in the spike protein that raise concerns of both antibody evasion and enhanced transmission. The B.1.351/Beta variant was found to be highly refractory to antibody neutralization ${ }^{4}$ and therefore compromised the efficacy of vaccines ${ }^{5-7}$ and therapeutic antibodies. The B.1.1.7/Alpha variant became dominant globally in early 2021 due to having an edge in transmission ${ }^{8}$, only to be replaced by the B.1.617.2/Delta variant, which exhibited an even greater propensity to spread coupled with a moderate level of antibody resistance ${ }^{9}$. Next came the B.1.1.529/Omicron variant, which was first detected in southern Africa in November 2021 (refs. $^{3,10,11}$ ) (Fig. 1a). Omicron has since spread rapidly in the region, as well as to over 60 countries, gaining traction even in regions in which the Delta variant is prevalent. The short doubling time (2-3 days) of Omicron cases suggests that it could soon become dominant ${ }^{2}$. Moreover, its spike protein contains an alarming number of mutations ( $>30)$ (Fig. 1b and Extended Data Fig. 1), including at least 15 in the receptor-binding domain (RBD), the principal target of neutralizing antibodies. These extensive spike mutations raise the possibility that current vaccines and therapeutic antibodies would be greatly compromised. This concern is amplified by the findings that we now report.

\section{Serum neutralization of B.1.1.529}

We first examined the neutralizing activity of serum collected in spring 2020 from patients with COVID-19, who were presumably infected with the wild-type (WT) SARS-CoV-2 (9-120 days after symptoms) (Methods and Extended Data Table 1). Samples from ten individuals were tested for neutralization against both D614G (WT) and B.1.1.529 pseudoviruses. Although robust titres were observed against D614G, a significant drop ( $>32$-fold) in $50 \%$ infectious dose $\left(\mathrm{ID}_{50}\right)$ titres was observed against B.1.1.529, with only two samples showing titres above the limit of detection (LOD) (Fig. 1c and Extended Data Fig. 2a). We next assessed the neutralizing activity of sera from individuals who received one of the four widely used COVID-19 vaccines: BNT162b2 (Pfizer, 15-213 days after vaccination), mRNA-1273 (Moderna, 6-177 days after vaccination), Ad26.COV2.S (Johnson \& Johnson, 50-186 days after vaccination) and ChAdOx1 nCoV-19 (AstraZeneca, 91-159

${ }^{1}$ Aaron Diamond AIDS Research Center, Columbia University Vagelos College of Physicians and Surgeons, New York, NY, USA. ${ }^{2}$ Department of Microbiology and Immunology, Columbia

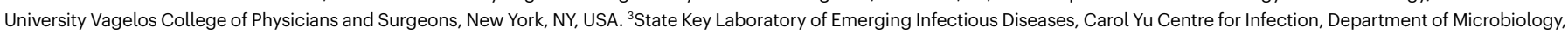
Li Ka Shing Faculty of Medicine, The University of Hong Kong, Hong Kong Special Administrative Region, China. ${ }^{4}$ Centre for Virology, Vaccinology and Therapeutics, Hong Kong Special Administrative Region, China. ${ }^{5}$ Department of Systems Biology, Columbia University Vagelos College of Physicians and Surgeons, New York, NY, USA. ${ }^{6}$ Division of Infectious Diseases,

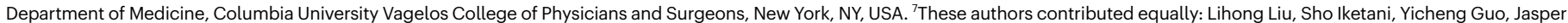
F.-W. Chan, Maple Wang, Liyuan Liu. ${ }^{凶}$-mail: dh2994@cumc.columbia.edu 

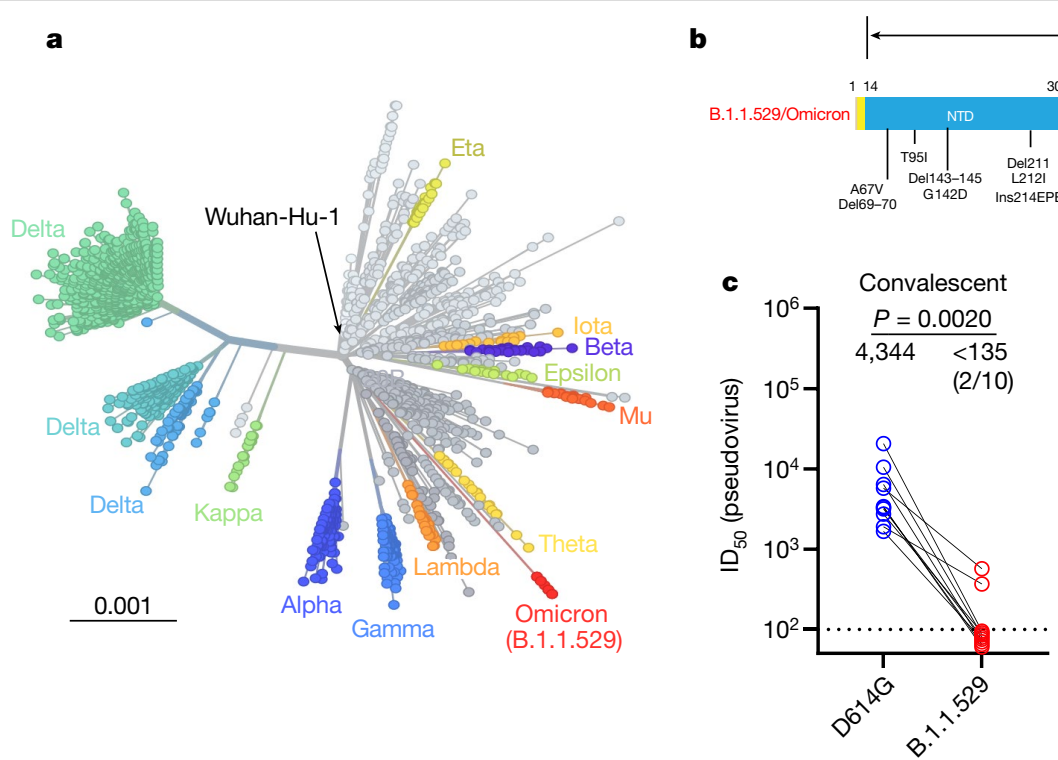

d

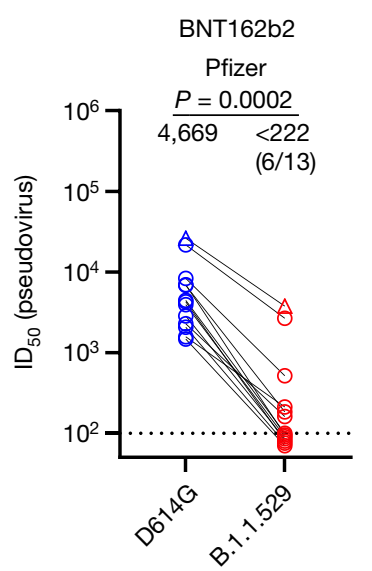

mRNA-1273

Moderna

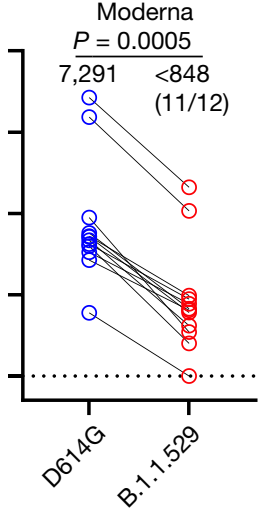

Fig.1 Resistance of B.1.1.529 to neutralization by sera. a, Unrooted phylogenetic tree of B.1.1.529 with other major SARS-CoV-2 variants. b, Key spike mutations found in the viruses isolated in the major lineage of B.1.1.529. Del, deletion; ins, insertion. c, Neutralization of D614G and B.1.1.529 pseudoviruses by convalescent patient sera.d, Neutralization of D614G and B.1.1.529 pseudoviruses by vaccinee sera. Within the four standard vaccination groups, individuals who were vaccinated without documented infection are denoted as circles and individuals who were both vaccinated and infected are denoted as
Ad26.Cov2.S Johnson \& Johnson

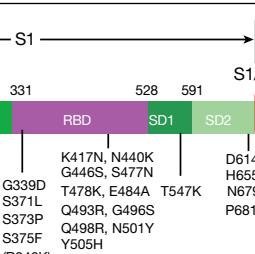
$\begin{array}{ll}\text { S373P } & \text { Q493R, G496S } \\ \text { S335F } & \text { Y488R, N501Y } \\ \text { (R346K) } & \end{array}$

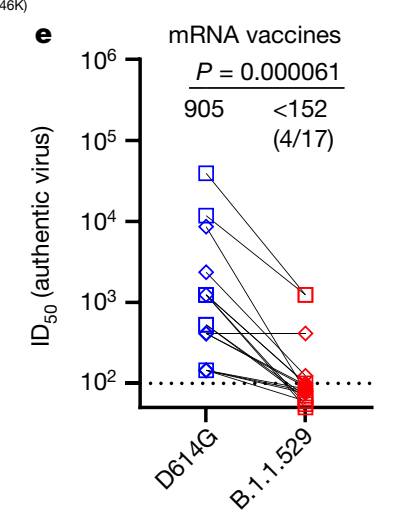

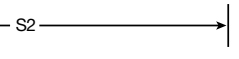

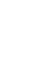

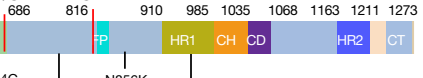
$\prod_{\substack{\text { Q954H } \\ \text { N969K }}}$ L981F

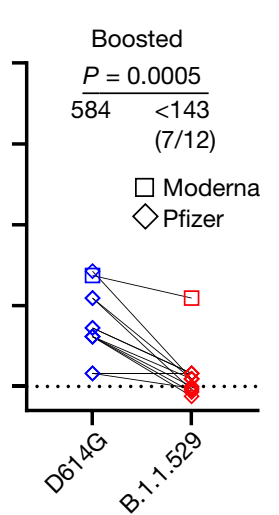

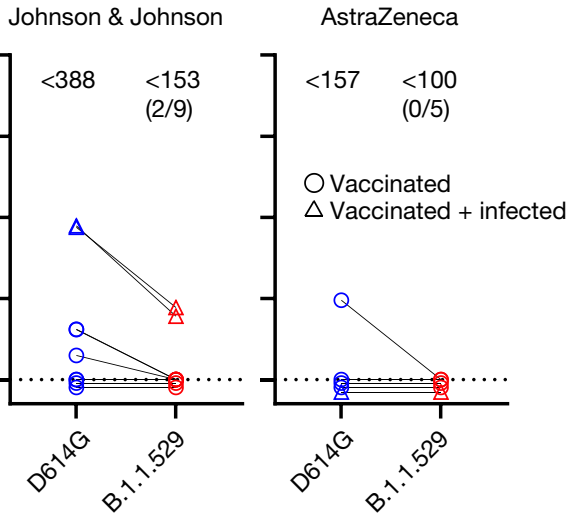

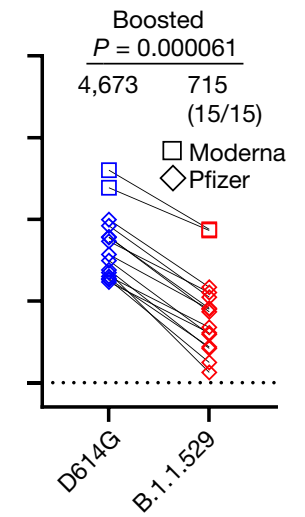

triangles. Within the boosted group, Moderna vaccinees are denoted as squares and Pfizer vaccinees are denoted as diamonds. e, Neutralization of authentic D614G and B.1.1.529 viruses by vaccinee sera. Moderna vaccinees are denoted as squares and Pfizer vaccinees are denoted as diamonds. Data represent one out of two independent experiments. For all of the panels, the values above the symbols denote the geometric mean titre and the numbers in parentheses denote the number of samples above the limit of detection. $P$ values were determined by using two-tailed Wilcoxon matched-pairs signed-rank tests.

days after vaccination) (Methods and Extended Data Table 2). In all cases, a substantial loss in neutralizing potency was observed against B.1.1.529 (Fig. 1d and Extended Data Fig. 2b-f). For the two mRNA-based vaccines, BNT162b2 and mRNA-1273, $a>21$-fold and $>8$.6-fold decrease in $\mathrm{ID}_{50}$ was observed, respectively. Note that, for these two groups, we specifically chose samples with high titres so that the fold change in titre could be better quantified; the difference in the number of samples with titres above the LOD ( 6 out of 13 for BNT162b2 versus 11 out of 12 for mRNA-1273) may therefore be favourably biased. Within the Ad26. COV2.S and ChAdOx1 nCOV-19 groups, all of the samples were below the LOD against B.1.1.529, except for two Ad26.COV2.S samples from patients who had a previous history of SARS-CoV-2 infection (Fig. 1d). Collectively, these results suggest that individuals who were previously infected or fully vaccinated remain at risk for B.1.1.529 infection.

Booster shots are now routinely administered in many countries 6 months after full vaccination. We therefore also examined the serum neutralizing activity of individuals who had received three homologous mRNA vaccinations (13 with BNT162b2 and 2 with mRNA-1273,
14-90 days after vaccination). Every sample showed lower activity in neutralizing B.1.1.529, with a mean decrease of 6.5-fold compared with the WT (Fig. 1d). Although all of the samples had titres above the LOD, the substantial loss in activity may still pose a risk for B.1.1.529 infection despite the booster vaccination.

We next confirmed the above findings by testing a subset of the BNT162b2 and mRNA-1273 vaccinee serum samples using authentic SARS-CoV-2 isolates:WT and B.1.1.529. Again, a substantial decrease in the neutralization of B.1.1.529 was observed, with mean decreases of $>6.0$-fold and $>4.1$-fold for the fully vaccinated group and the boosted group, respectively (Fig. 1e).

\section{Antibody neutralization of B.1.1.529}

To understand the types of antibodies in the serum that lost neutralizing activity against B.1.1.529, we assessed the neutralization profile of 19 well-characterized monoclonal antibodies against the spike protein, including 17 directed to the RBD and 2 directed to the $\mathrm{N}$-terminal 


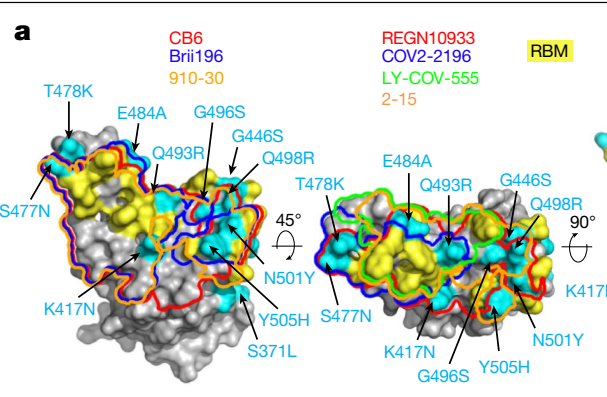

Class 1

Class 2

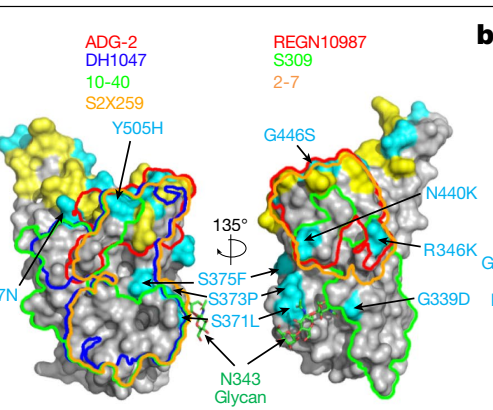

Class 4

Class 3

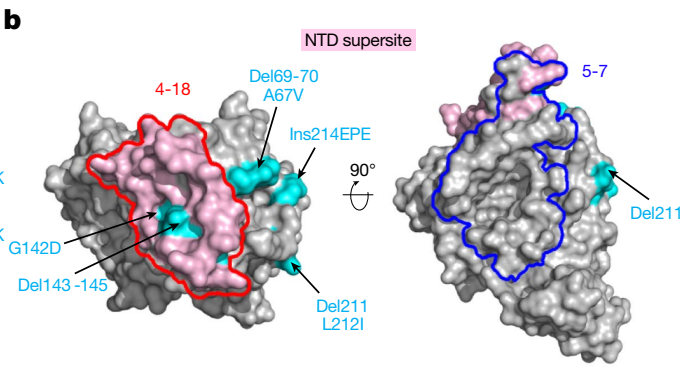

NTD supersite view

Alternative site view

c
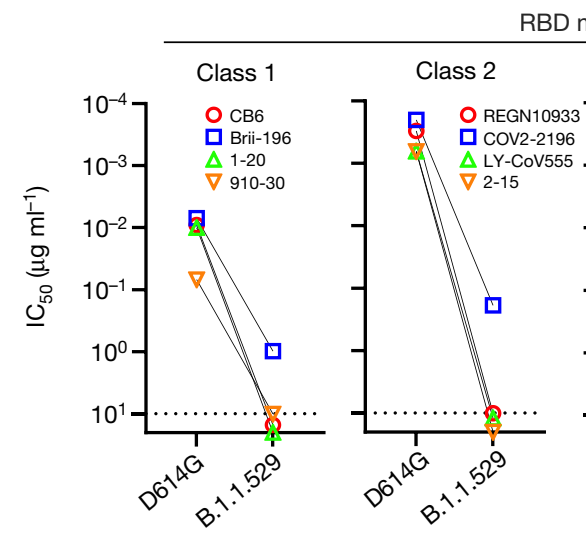

RBD mAbs

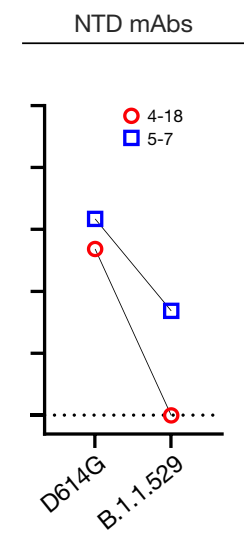

Combinations

d

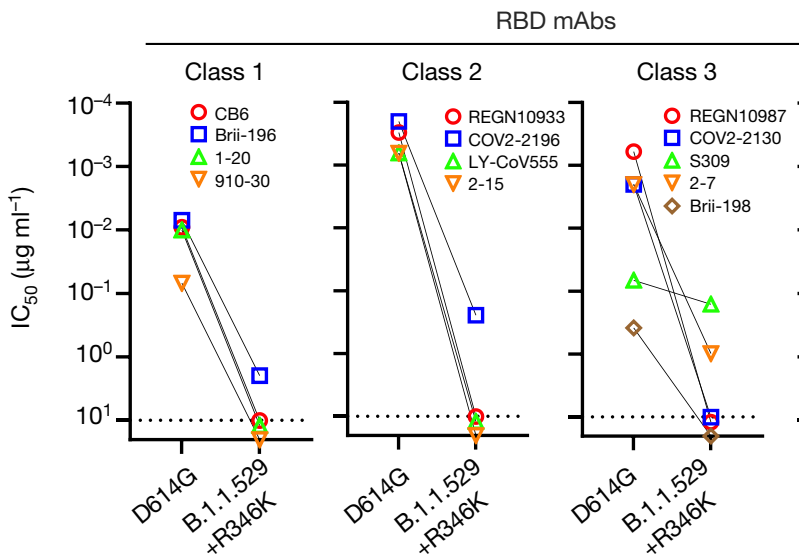

Fig. 2 | Resistance of B.1.1.529 to neutralization by monoclonal antibodies. a, Footprints of RBD-directed antibodies. Mutations within B.1.1.529 are highlighted in cyan. Approved or authorized antibodies are shown in bold. The receptor-binding motif (RBM) residues are highlighted in yellow. b, Footprints of NTD-directed antibodies. Mutations within B.1.1.529 are highlighted in cyan. The NTD supersite residues are highlighted in light pink.

domain (NTD). We included the following monoclonal antibodies that have been authorized or approved for clinical use, either individually or in combination: REGN10987 (imdevimab) ${ }^{12}$, REGN10933 (casirivimab) ${ }^{12}$, COV2-2196 (tixagevimab) ${ }^{13}$, COV2-2130 (cilgavimab) ${ }^{13}$, LY-CoV555 (bamlanivimab $)^{14}$, CB6 (etesevimab) ${ }^{15}$, Brii-196 (amubarvimab) ${ }^{16}$, Brii-198 (romlusevimab) ${ }^{16}$ and S309 (sotrovimab) $)^{17}$. We also included other monoclonal antibodies of interest: $910-30$ (ref. ${ }^{18}$ ), ADG-2 (ref. ${ }^{19}$ ), DH1047 (ref. ${ }^{20}$ ), S2X259 (ref. $^{21}$ ) and our antibodies 1-20, 2-15, 2-7, 4-18, 5-7 and 10-40 (refs. ${ }^{22-24}$ ). The footprints of monoclonal antibodies with structures available were drawn in relation to the mutations found in the B.1.1.529 RBD (Fig. 2a) and NTD (Fig. 2b). The risk to each of the four classes $^{25}$ of RBD monoclonal antibodies, as well as to the NTD monoclonal antibodies, was immediately apparent. Indeed, neutralization studies on B.1.1.529 pseudovirus showed that 17 out of the 19 monoclonal antibodies tested lost neutralizing activity completely or partially

(Fig. 2c and Extended Data Fig. 3). The potency of class 1 and class 2 RBD monoclonal antibodies all decreased by more than 100 -fold, as did the more potent monoclonal antibodies in RBD class 3 (REGN10987, COV2-2130 and 2-7). The activities of S309 and Brii-198 were spared. All of the monoclonal antibodies in RBD class 4 lost neutralization potency against B.1.1.529 by at least tenfold, as did monoclonal antibodies directed to the antigenic supersite ${ }^{26}(4-18)$ or the alternative site $^{23}$ (5-7) on the NTD. Strikingly, all four combination monoclonal antibody drugs in clinical use lost substantial activity against B.1.1.529, probably abolishing or impairing their efficacy in patients.

Approximately $10 \%$ of the B.1.1.529 viruses in the Global Initiative on Sharing All Influenza Data (GISAID) ${ }^{1}$ also contain an additional RBD mutation, $\mathrm{R} 346 \mathrm{~K}$, which is the defining mutation for the B.1.621/Mu vari$\mathrm{ant}^{27}$. We therefore constructed another pseudovirus (B.1.1.529+R346K) containing this mutation for additional testing using the same panel 


\begin{tabular}{|c|c|c|c|c|c|c|c|c|c|c|c|c|c|c|c|c|c|c|c|}
\hline \multirow{3}{*}{$\begin{array}{l}\text { Fold change in } \\
\mathrm{IC}_{50} \text { compared } \\
\text { with WT }\end{array}$} & \multicolumn{17}{|c|}{ RBD mAbs } & \multirow{2}{*}{\multicolumn{2}{|c|}{ NTD mAbs }} \\
\hline & \multicolumn{4}{|c|}{ Class 1} & \multicolumn{4}{|c|}{ Class 2} & \multicolumn{5}{|c|}{ Class 3} & \multicolumn{4}{|c|}{ Class 4} & & \\
\hline & СB6 & Brit-196 & 1-20 & $910-30$ & REGN10933 & Cov2-2196 & LY-Cov555 & 2-15 & REGN10987 & cov2-2130 & s309 & $2-7$ & Brii-198 & ADG-2 & DH1047 & $10-40$ & $\mathrm{~s} 2 \times 259$ & $4-18$ & $5-7$ \\
\hline B.1.1.529+R346K & $<-761$ & -97 & $<-338$ & $<-159$ & $<-1,000$ & -89 & $<-1,000$ & $<-1,000$ & $<-1,000$ & $<-988$ & -2.4 & -109 & $<-32$ & -51 & -167 & -32 & -16 & -125 & -33 \\
\hline A67V & 1.1 & 1.0 & -1.1 & 1.4 & 1.1 & -1.0 & 1.1 & 1.1 & 1.1 & 1.2 & -1.4 & -1.1 & -1.2 & 1.3 & -1.3 & -1.1 & 1.0 & -1.6 & -1.1 \\
\hline Del69-70 & -1.4 & -1.4 & -1.6 & -1.1 & -1.8 & -1.5 & -1.4 & -1.4 & -1.7 & -1.4 & -2.2 & -1.9 & -2.3 & -1.4 & -3.3 & -1.7 & -1.3 & -2.6 & -9.4 \\
\hline Del211 & -2.4 & -2.1 & $\begin{array}{l}-1.6 \\
\end{array}$ & -2.1 & -1.5 & -1.5 & -1.4 & -1.2 & 1.2 & -1.2 & -1.2 & -1.3 & -1.1 & -1.9 & -2.4 & -1.6 & -2.3 & 1.2 & -9.1 \\
\hline L212I & -1.3 & -1.8 & -1.3 & -1.6 & -1.4 & -1.4 & -1.6 & -1.3 & -1.3 & -1.4 & -2.2 & -1.9 & -2.2 & -1.7 & -3.2 & -2.0 & -1.9 & -7.2 & -2.2 \\
\hline Ins214EPE & -2.4 & -2.4 & -2.2 & -2.4 & -2.8 & -2.7 & -2.3 & -4.3 & -3.0 & -2.2 & -3.0 & -6.2 & -2.7 & -3.1 & -2.9 & -1.9 & -3.3 & -7.1 & -15 \\
\hline G339D & -1.7 & -1.6 & -1.7 & -1.4 & -2.2 & -1.7 & -1.5 & -1.4 & -1.8 & -1.6 & -4.0 & -1.9 & -3.9 & -1.6 & -2.2 & -1.5 & -3.2 & -4.5 & -3.0 \\
\hline R346K & -1.5 & -1.2 & -1.3 & 1.0 & -1.5 & -1.3 & -1.3 & -1.4 & -1.6 & -2.9 & -1.4 & -1.0 & -21 & -1.1 & -1.9 & -1.2 & -1.4 & -1.4 & -2.3 \\
\hline S371L & -19 & -18 & -15 & -22 & -10 & -4.1 & -2.9 & -1.4 & -25 & -1.4 & -12 & -12 & -17 & -18 & -49 & -59 & -23 & -1.8 & 1.1 \\
\hline S373P & -1.9 & -2.1 & -1.6 & -1.4 & -1.9 & -2.1 & -2.0 & -1.4 & -1.9 & -1.3 & -2.3 & -1.8 & -2.5 & -2.2 & -5.1 & -5.0 & -2.8 & -8.2 & -5.0 \\
\hline T478K & 1.2 & 1.1 & 1.4 & 1.6 & 1.3 & -1.5 & -1.4 & -1.2 & -1.6 & 1.1 & -1.8 & -2.6 & -1.6 & -1.2 & -2.8 & -1.3 & -2.3 & -3.3 & -2.3 \\
\hline E484A & -2.8 & -1.7 & $\begin{array}{l}-1.8 \\
\end{array}$ & -1.2 & -4.8 & -4.9 & $<-1,000$ & \begin{tabular}{|l|l|}
$<-000$ \\
\end{tabular} & -1.6 & -1.4 & -1.4 & -2.7 & -1.9 & -1.6 & -1.5 & \begin{tabular}{|l|}
-1.9 \\
\end{tabular} & $\begin{array}{l}-1.9 \\
\end{array}$ & -5.7 & -2.9 \\
\hline Q493R & -16 & -7.3 & -3.2 & 2.9 & -42 & -4.2 & $<-1,000$ & -705 & -1.4 & -1.1 & -1.2 & -1.9 & $\begin{array}{l}-2.0 \\
\end{array}$ & $\begin{array}{l}-1.6 \\
\end{array}$ & -1.6 & $\begin{array}{l}-1.6 \\
\end{array}$ & -1.5 & -4.0 & -1.3 \\
\hline G496S & -1.3 & 1.3 & 1.1 & 1.1 & 1.0 & 1.1 & 1.0 & -9.3 & -6.2 & -1.3 & -1.4 & 1.4 & -1.2 & -1.2 & -1.6 & -1.1 & -1.6 & -2.6 & -1.6 \\
\hline Q498R & -1.7 & -1.2 & 1.1 & 1.4 & -1.5 & -1.1 & -1.4 & -1.0 & -1.6 & -1.4 & -1.3 & 1.1 & -1.2 & 2.4 & -1.3 & -1.2 & -1.3 & -1.5 & -1.8 \\
\hline N501Y & -9.8 & -1.2 & -8.4 & -16 & -1.4 & -1.5 & -1.6 & -1.2 & -1.2 & -1.1 & $\begin{array}{l}-1.8 \\
\end{array}$ & -1.5 & -2.7 & -1.8 & -2.5 & -1.9 & -1.9 & -20 & -3.9 \\
\hline $\mathrm{Y} 505 \mathrm{H}$ & -1.2 & 1.2 & -1.3 & -9.6 & 1.1 & 1.0 & 1.0 & 1.1 & 1.4 & 1.0 & -1.4 & 1.7 & 1.1 & 1.3 & -1.4 & 1.0 & -1.2 & -1.2 & -1.1 \\
\hline T547K & -1.9 & -2.0 & -2.0 & -1.9 & -1.7 & -1.3 & -1.6 & -1.7 & -2.7 & -1.6 & -1.6 & -4.3 & $\begin{array}{l}-1.9 \\
\end{array}$ & $\begin{array}{l}-1.7 \\
\end{array}$ & -2.6 & -1.5 & -1.9 & -2.7 & -2.7 \\
\hline $\mathrm{H} 655 \mathrm{Y}$ & -2.7 & -3.1 & -3.5 & -2.7 & -3.1 & -2.0 & -2.2 & -8.6 & -8.8 & -1.7 & -2.3 & -13 & -2.4 & -2.1 & -3.9 & -3.3 & -3.9 & -23 & -5.3 \\
\hline N679K & 1.0 & 1.2 & 1.1 & 1.1 & -1.1 & -1.2 & -1.2 & -1.2 & -1.9 & -1.1 & -1.3 & -1.8 & -1.7 & -1.4 & -2.4 & -1.7 & -1.7 & -2.1 & -2.7 \\
\hline $\mathrm{P} 681 \mathrm{H}$ & -2.3 & -2.1 & -2.1 & 1.0 & -2.4 & -1.8 & -2.2 & -1.5 & -1.5 & -1.0 & -1.6 & -1.9 & -1.5 & -1.3 & -2.3 & -1.3 & -1.3 & -2.3 & -2.4 \\
\hline N764K & -1.1 & -1.5 & -1.3 & -1.1 & -1.4 & -1.4 & -1.4 & -2.1 & -2.5 & -1.5 & -2.2 & -4.3 & $\begin{array}{l}-1.3 \\
\end{array}$ & $\begin{array}{l}-1.4 \\
\end{array}$ & -3.3 & -2.1 & -2.4 & -2.3 & -2.1 \\
\hline D769Y & 1.3 & 1.1 & 1.0 & 1.2 & -1.5 & -1.0 & -1.4 & -1.4 & -2.0 & -1.3 & -1.9 & -2.5 & -1.3 & -1.1 & -1.7 & -1.2 & -1.4 & -3.1 & -2.5 \\
\hline N856K & -10 & -2.8 & -1.3 & -12 & -2.2 & -3.0 & -1.1 & -1.0 & -1.4 & -1.1 & -1.2 & -1.3 & -2.3 & -1.8 & -4.4 & -2.1 & -2.5 & -1.6 & -1.9 \\
\hline Q954H & 2.7 & 1.9 & 1.5 & 2.6 & 1.2 & 1.0 & 1.2 & 1.1 & -1.1 & 1.2 & -1.2 & -1.4 & -1.1 & -1.1 & -2.5 & -1.0 & -1.1 & -2.3 & -2.9 \\
\hline
\end{tabular}

b
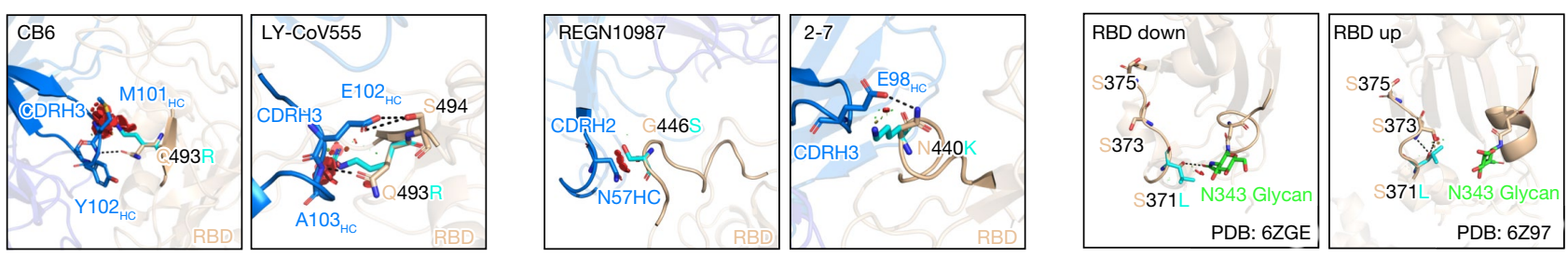

Fig. 3 | Impact of individual mutations within B.1.1.529 against monoclonal antibodies. a, Neutralization of pseudoviruses containing single mutations found within B.1.1.529 by a panel of 19 monoclonal antibodies. The fold change relative to neutralization of D614G is denoted, with resistance coloured red and sensitization coloured green. b, Modelling of critical mutations in B.1.1.529 that affect antibody neutralization. of monoclonal antibodies (Fig. 2d). The overall findings resembled those already shown in Fig. $2 \mathrm{c}$, with the exception that the neutralizing activity of Brii-198 was abolished. In fact, nearly the entire panel of antibodies was essentially rendered inactive against this minor form of the Omicron variant.

The fold changes in $\mathrm{IC}_{50}$ of the monoclonal antibodies against B.1.1.529 and B.1.1.529+R346K relative to D614G are summarized in the first two rows of Fig. 3a. The considerable loss of activity observed for all classes of monoclonal antibodies against B.1.1.529 suggests that perhaps the same is occurring in the serum of convalescent patients and vaccinated individuals.

\section{Mutations conferring antibody resistance}

To understand the specific B.1.1.529 mutations that confer antibody resistance, we next individually tested the same panel of 19 monoclonal antibodies against pseudoviruses for each of the 34 mutations (excluding D614G) found in B.1.1.529 or B.1.1.529+R346K. Our findings not only confirmed the role of known mutations at spike residues 142-145, 417, 484 and 501 in conferring resistance to NTD or RBD (class 1 or class 2 ) antibodies ${ }^{4}$ but also revealed several mutations that were not previously known to have functional importance to neutralization (Fig. 3a and Extended Data Fig. 4). Q493R, which was previously shown to affect binding of CB6 and LY-CoV555 (ref. ${ }^{28}$ ) as well as polyclonal sera ${ }^{29}$, mediated resistance to CB6 (class 1 ) as well as to LY-CoV555 and 2-15 (class 2), findings that could be explained by the abolishment of hydrogen bonds due to the long side chain of arginine and induced steric clashes with CDRH 3 in these antibodies (Fig. 3b (left)). Both N440K and G446S mediated resistance to REGN10987 and 2-7 (class 3), observations that could also be explained by steric hindrance (Fig. 3b (middle)). Themoststriking and perhaps unexpected finding wasthat S371Lbroadly affected neutralization by monoclonal antibodies in all four RBD classes (Fig. 3a and Extended Data Fig. 4). Although the precise mechanism of this resistance is unknown, in silico modelling suggested two possibilities (Fig. 3b (right)). First, in the RBD-down state, mutating Ser to Leu results in an interference with the $\mathrm{N} 343$ glycan, thereby possibly altering 


\begin{tabular}{|c|c|c|c|c|c|c|c|c|c|c|c|c|c|c|c|c|c|c|c|}
\hline \multirow{3}{*}{$\begin{array}{l}\text { Fold change in } \\
I_{50} \text { compared } \\
\text { with WT }\end{array}$} & \multicolumn{17}{|c|}{ RBD mAbs } & \multirow{2}{*}{\multicolumn{2}{|c|}{ NTD mAbs }} \\
\hline & \multicolumn{4}{|c|}{ Class 1} & \multicolumn{4}{|c|}{ Class 2} & \multicolumn{5}{|c|}{ Class 3} & \multicolumn{4}{|c|}{ Class 4} & & \\
\hline & CB6 & Brii-196 & $1-20$ & $910-30$ & REGN10933 & Cov2-2196 & LY-Cov555 & $2-15$ & REGN10987 & cov2-2130 & s309 & $2-7$ & Brii-198 & ADG-2 & $\begin{array}{l}\text { DH1047 } \\
\end{array}$ & $10-40$ & $\mathrm{~s} 2 \times 259$ & $4-18$ & 5-7 \\
\hline B.1.1.7 & -8.8 & 2.6 & -5.2 & -15 & 1.6 & 1.8 & 1.6 & 2.2 & 2.9 & 1.7 & 1.1 & 2.3 & 4.1 & 1.7 & 2.2 & 1.4 & 1.4 & -5.1 & -4.0 \\
\hline B.1.526 & -1.0 & 1.1 & -1.1 & 2.5 & -4.5 & -2.1 & -590 & $-1,329$ & 1.8 & 1.2 & 2.9 & 1.8 & -1.1 & 1.5 & 2.9 & -2.2 & 1.4 & 4.5 & -2.5 \\
\hline B.1.429 & 3.0 & 2.3 & 1.4 & 2.5 & 2.5 & 2.8 & -590 & -4.6 & 1.6 & 1.1 & 1.9 & 1.6 & -2.4 & 2.0 & 2.9 & 1.3 & 3.3 & -39 & -59 \\
\hline B.1.617.2 & 2.1 & 1.2 & -1.1 & 2.5 & 1.2 & 1.4 & -590 & -10 & -1.8 & -1.7 & 1.2 & -1.1 & -8.9 & 1.0 & 1.4 & -1.8 & -1.4 & -39 & -74 \\
\hline P.1 & -196 & 2.2 & -16 & -60 & -121 & -2.0 & -590 & $-1,329$ & 1.9 & 1.1 & 1.1 & 1.2 & 1.8 & -1.0 & 3.0 & -2.2 & 1.2 & -39 & -74 \\
\hline B.1.351 & -196 & 2.0 & -40 & -60 & -78 & -2.5 & -590 & $-1,329$ & 1.5 & 1.5 & 1.2 & 1.9 & -1.5 & 1.0 & 3.0 & -2.9 & 1.2 & -39 & -8.4 \\
\hline B.1.1.529 & $<-1,000$ & -134 & $<-338$ & $<-159$ & $<-1,000$ & -140 & $<-1,000$ & $<-1,000$ & $<-1,000$ & -390 & -2.5 & -231 & 2.2 & -43 & -124 & -11 & -35 & -125 & -30 \\
\hline B.1.1.529+R346K & $<-761$ & -97 & $<-338$ & $<-159$ & $<-1,000$ & -89 & $<-1,000$ & $<-1,000$ & $<-1,000$ & $<-988$ & -2.4 & -109 & $<-32$ & -51 & -167 & -32 & -16 & -125 & -33 \\
\hline
\end{tabular}

Fig. 4 | Evolution of antibody resistance across SARS-CoV-2 variants. Neutralization of SARS-CoV-2 variant pseudoviruses by a panel of 19 monoclonal antibodies. The fold change relative to neutralization of D614G is denoted.

its conformation and affecting class 3 antibodies that typically bind to this region. Second, in the RBD-up state, S371L may alter the local conformation of the loop consisting of Ser371-Ser373-Ser375, thereby affecting the binding of class 4 antibodies that generally target a portion of this loop ${ }^{24}$. It is not clear how class 1 and class 2 RBD monoclonal antibodies are affected by this mutation.

\section{Evolution of SARS-CoV-2 to antibodies}

To gain insights into the antibody resistance of B.1.1.529 relative to previous SARS-CoV-2 variants, we evaluated the neutralizing activity of the same panel of neutralizing monoclonal antibodies against pseudoviruses for B.1.1.7 (ref. $^{8}$ ), B.1.526 (ref. ${ }^{30}$ ), B.1.429 (ref. ${ }^{31}$ ), B.1.617.2 (ref. ${ }^{9}$ ), P.1 (ref. ${ }^{32}$ ) and B.1.351 (ref. ${ }^{33}$ ). It is evident from these results (Fig. 4 and Extended Data Fig. 5) that previous variants developed resistance to only NTD antibodies and class 1 and class 2 RBD antibodies. Here B.1.1.529, with or without R346K, has made a big mutational leap by becoming not only nearly completely resistant to class 1 and class 2 RBD antibodies, but also substantially resistant to both class 3 and class 4 RBD antibodies. B.1.1.529 is now the most complete 'escapee' from neutralization by currently available antibodies.

\section{Discussion}

The Omicron variant caused fear almost as soon as it was detected to be spreading in South Africa. The suggestion that this new variant would transmit more readily has come true in the ensuing weeks ${ }^{2}$. The extensive mutations found in its spike protein raised concerns that the efficacy of current COVID-19 vaccines and antibody therapies might be compromised. Indeed, in this study, sera from convalescent patients (Fig. 1c) and vaccinees (Fig. 1d, e) showed markedly reduced neutralizing activity against B.1.1.529. Other studies have found similar losses $^{34-38}$. These findings are consistent with emerging clinical data on the Omicron variant demonstrating higher rates of reinfection ${ }^{11}$ and vaccine breakthroughs. In fact, recent reports showed that the efficacy of two doses of BNT162b2 vaccine has dropped from over 90\% against the original SARS-CoV-2 strain to approximately $40 \%$ and 33\% against B.1.1.529 in the United Kingdom ${ }^{39}$ and South Africa ${ }^{40}$, respectively. Even a third booster shot may not adequately protect against Omicron infection ${ }^{39,41}$, although the protection against disease still makes it advisable to administer booster vaccinations. Vaccines that elicited lower neutralizing titres ${ }^{35,42}$ are expected to fare worse against B.1.1.529.

The nature of the loss in serum neutralizing activity against B.1.1.529 could be discerned from our findings on a panel of monoclonal antibodies directed to the viral spike. The neutralizing activities of all four major classes of RBD monoclonal antibodies and two distinct classes of NTD monoclonal antibodies are either abolished or impaired (Fig. 2c, d). In addition to previously identified mutations that confer antibody resistance ${ }^{4}$, we have uncovered four previously undescribed spike mutations with functional consequences. Q493R confers resistance to some class 1 and class 2 RBD monoclonal antibodies; N440K and G446S confer resistance to some class 3 RBD monoclonal antibodies; and S371L confers global resistance to many RBD monoclonal antibodies through mechanisms that are not yet apparent. While performing these monoclonal antibody studies, we also observed that nearly all of the currently authorized or approved monoclonal antibody drugs are rendered weak or inactive by B.1.1.529 (Figs. 2c and 3a). In fact, the Omicron variant that contains $\mathrm{R} 346 \mathrm{~K}$ renders almost all current antibody therapy for COVID-19 ineffective (Figs. 2d and 3a).

The scientific community has chased after SARS-CoV-2 variants for a year. As more and more of them appeared, our interventions directed to the spike became increasingly ineffective. The Omicron variant has now put an exclamation mark on this point. It is not too farfetched to think that this SARS-CoV-2 is now only a mutation or two away from being pan-resistant to current antibodies, either monoclonal or polyclonal. We must devise strategies that anticipate the evolutional direction of the virus and develop agents that target better-conserved viral elements.

\section{Online content}

Any methods, additional references, Nature Research reporting summaries, source data, extended data, supplementary information, acknowledgements, peer review information; details of author contributions and competing interests; and statements of data and code availability are available at https://doi.org/10.1038/s41586-021-04388-0.

1. Shu, Y. \& McCauley, J. GISAID: global initiative on sharing all influenza data-from vision to reality. Euro Surveill. 22, 30494 (2017).

2. Grabowski, F., Kochańczyk, M. \& Lipniacki, T. Omicron strain spreads with the doubling time of 3.2-3.6 days in South Africa province of Gauteng that achieved herd immunity to Delta variant. Preprint at https://doi.org/10.1101/2021.12.08.21267494 (2021).

3. Scott, L. et al. Track Omicron's spread with molecular data. Science 374, 1454-1455 (2021).

4. Wang, P. et al. Antibody resistance of SARS-CoV-2 variants B.1.351 and B.1.1.7. Nature 593, 130-135 (2021).

5. Abu-Raddad, L. J., Chemaitelly, H. \& Butt, A. A. Effectiveness of the BNT162b2 COVID-19 vaccine against the B.1.1.7 and B.1.351 variants. N. Engl. J. Med. 385, 187-189 (2021).

6. Madhi, S. A. et al. Efficacy of the ChAdOx1 nCoV-19 COVID-19 vaccine against the B.1.351 variant. N. Engl. J. Med. 384, 1885-1898 (2021).

7. Sadoff, J. et al. Safety and efficacy of single-dose Ad26.COV2.S vaccine against COVID-19. N. Engl. J. Med. 384, 2187-2201 (2021).

8. Davies, N. G. et al. Estimated transmissibility and impact of SARS-CoV-2 lineage B.1.1.7 in England. Science 372, eabg3055 (2021).

9. Mlcochova, P. et al. SARS-CoV-2 B.1.617.2 Delta variant replication and immune evasion. Nature 599, 114-119 (2021).

10. Hadfield, J. et al. Nextstrain: real-time tracking of pathogen evolution. Bioinformatics $\mathbf{3 4}$, 4121-4123 (2018).

11. Pulliam, J. R. C. et al. Increased risk of SARS-CoV-2 reinfection associated with emergence of the Omicron variant in South Africa. Preprint at https://doi. org/10.1101/2021.11.11.21266068 (2021).

12. Hansen, J. et al. Studies in humanized mice and convalescent humans yield a SARS-CoV-2 antibody cocktail. Science 369, 1010-1014 (2020).

13. Zost, S. J. et al. Potently neutralizing and protective human antibodies against SARS-CoV-2. Nature 584, 443-449 (2020). 
14. Jones, B. E. et al. The neutralizing antibody, LY-CoV555, protects against SARS-CoV-2 infection in nonhuman primates. Sci. Transl. Med. 13, eabf1906 (2021).

15. Shi, R. et al. A human neutralizing antibody targets the receptor-binding site of SARS-CoV-2. Nature 584, 120-124 (2020).

16. Ju, B. et al. Human neutralizing antibodies elicited by SARS-CoV-2 infection. Nature 584, $115-119$ (2020).

17. Pinto, D. et al. Cross-neutralization of SARS-CoV-2 by a human monoclonal SARS-CoV antibody. Nature 583, 290-295 (2020).

18. Banach, B. B. et al. Paired heavy- and light-chain signatures contribute to potent SARS-CoV-2 neutralization in public antibody responses. Cell Rep 37, 109771 (2021).

19. Rappazzo, C. G. et al. Broad and potent activity against SARS-like viruses by an engineered human monoclonal antibody. Science 371, 823-829 (2021).

20. Li, D. et al. In vitro and in vivo functions of SARS-CoV-2 infection-enhancing and neutralizing antibodies. Cell 184, 4203-4219 (2021).

21. Tortorici, M. A. et al. Broad sarbecovirus neutralization by a human monoclonal antibody. Nature 597, 103-108 (2021).

22. Liu, L. et al. Potent neutralizing antibodies against multiple epitopes on SARS-CoV-2 spike. Nature 584, 450-456 (2020).

23. Cerutti, G. et al. Neutralizing antibody 5-7 defines a distinct site of vulnerability in SARS-CoV-2 spike N-terminal domain. Cell Rep. 37, 109928 (2021).

24. Liu, L. et al. Isolation and comparative analysis of antibodies that broadly neutralize sarbecoviruses. Preprint at https://doi.org/10.1101/2021.12.11.472236 (2021).

25. Barnes, C. O. et al. SARS-CoV-2 neutralizing antibody structures inform therapeutic strategies. Nature 588, 682-687 (2020).

26. Cerutti, G. et al. Potent SARS-CoV-2 neutralizing antibodies directed against spike $\mathrm{N}$-terminal domain target a single supersite. Cell Host Microbe 29, 819-833 (2021).

27. Uriu, K. et al. Neutralization of the SARS-CoV-2 Mu variant by convalescent and vaccine serum. N. Engl. J. Med. 385, 2397-2399 (2021).

28. Starr, T. N., Greaney, A. J., Dingens, A. S. \& Bloom, J. D. Complete map of SARS-CoV-2 RBD mutations that escape the monoclonal antibody LY-CoV555 and its cocktail with LY-CoV016. Cell Rep. Med. 2, 100255 (2021).

29. Amanat, F. et al. SARS-CoV-2 mRNA vaccination induces functionally diverse antibodies to NTD, RBD, and S2. Cell 184, 3936-3948 (2021).
30. Annavajhala, M. K. et al. Emergence and expansion of SARS-CoV-2 B.1.526 after identification in New York. Nature 597, 703-708 (2021).

31. Zhang, W. et al. Emergence of a novel SARS-CoV-2 variant in southern California. JAMA 325, 1324-1326 (2021).

32. Faria, N. R. et al. Genomics and epidemiology of the P.1 SARS-CoV-2 lineage in Manaus, Brazil. Science 372, 815-821 (2021).

33. Tegally, H. et al. Detection of a SARS-CoV-2 variant of concern in South Africa. Nature 592, 438-443 (2021).

34. Schmidt, F. et al. Plasma neutralization of the SARS-CoV-2 Omicron variant. New Engl. J. Med. https://doi.org/10.1056/NEJMc2119641 (2021).

35. Garcia-Beltran, W. F. et al. mRNA-based COVID-19 vaccine boosters induce neutralizing immunity against SARS-CoV-2 Omicron variant. Cell, https://doi.org/10.1016/j.cell.2021.12.033 (2022).

36. Cameroni, E. et al. Broadly neutralizing antibodies overcome SARS-CoV-2 Omicron antigenic shift. Nature https://doi.org/10.1038/s41586-021-04386-2 (2021).

37. Doria-Rose, N. A. et al. Booster of mRNA-1273 vaccine reduces SARS-CoV-2 Omicron escape from neutralizing antibodies. Preprint at https://doi.org/10.1101/2021.12.15.21267805 (2021).

38. Planas, D. et al. Considerable escape of SARS-CoV-2 variant Omicron to antibody neutralization. Nature https://doi.org/10.1038/s41586-021-04389-z (2021).

39. Andrews, N. et al. Effectiveness of COVID-19 vaccines against the Omicron (B.1.1.529) variant of concern. Preprint at https://doi.org/10.1101/2021.12.14.21267615 (2021).

40. Wroughton, L. Omicron variant more resistant to vaccine but causes less severe covid, major South African study concludes. The Washington Post (14 December 2021).

41. Kuhlmann, C. et al. Breakthrough infections with SARS-CoV-2 Omicron variant despite booster dose of mRNA vaccine. SSRN https://doi.org/10.2139/ssrn.3981711 (2021).

42. Cromer, D. et al. Neutralising antibody titres as predictors of protection against SARS-CoV-2 variants and the impact of boosting: a meta-analysis. Lancet Microbe $\mathbf{3}$, e52-e61 (2021).

Publisher's note Springer Nature remains neutral with regard to jurisdictional claims in published maps and institutional affiliations.

(c) The Author(s), under exclusive licence to Springer Nature Limited 2021 


\section{Methods}

\section{Data reporting}

No statistical methods were used to predetermine sample size. The experiments were not randomized and the investigators were not blinded to allocation during experiments and outcome assessment.

\section{Serum samples}

Convalescent plasma samples were obtained from patients with documented SARS-CoV-2 infection. These samples were collected the beginning of the pandemic in early 2020 at Columbia University Irving Medical Center and are therefore assumed to be infection by the WT strain of SARS-CoV-2 $\left(\right.$ ref. $\left.^{4}\right)$. Sera from individuals who received two or three doses of mRNA-1273 or BNT162b2 vaccine were collected at Columbia University Irving Medical Center at least two weeks after the final dose. Sera from individuals who received one dose of Ad26. COV2.S or two doses of ChAdOx1 nCov-19 were obtained from BEI Resources. Some individuals were also infected by SARS-CoV-2 in addition to the vaccinations they received. Note that, whenever possible, we specifically chose samples with high titres against the WT strain of SARS-CoV-2 such that the loss in activity against B.1.1.529 could be better quantified and the titres observed here should therefore be considered in that context. All collections were conducted under protocols reviewed and approved by the Institutional Review Board of Columbia University. All of the participants provided written informed consent. Additional information for the convalescent samples and vaccinee samples is provided in Extended Data Tables 1 and 2 , respectively.

\section{Monoclonal antibodies}

Antibodies were expressed as previously described ${ }^{22}$ by synthesis of heavy chain variable $(\mathrm{VH})$ and light chain variable $(\mathrm{VL})$ genes (GenScript), transfection of Expi293 cells (Thermo Fisher Scientific) and affinity purification from the supernatant by rProtein A Sepharose (GE). REGN10987, REGN10933, COV2-2196 and COV2-2130 were provided by Regeneron Pharmaceuticals; Brii-196 and Brii-198 were provided by Brii Biosciences; CB6 was provided by B. Zhang and $\mathrm{P}$. Kwong (NIH); and 910-30 was provided by B. DeKosky (MIT).

\section{Cell lines}

Expi293 cells were obtained from Thermo Fisher Scientific (A14527); Vero E6 cells were obtained from the ATCC (CRL-1586); HEK293T cells were obtained from the ATCC (CRL-3216); and Vero-E6-TMPRSS2 cells were obtained from JCRB (JCRB1819). Cells were purchased from authenticated vendors and morphology was confirmed visually before use. All cell lines tested mycoplasma negative.

\section{Variant SARS-CoV-2 spike plasmid construction}

An in-house high-throughput template-guide gene synthesis approach was used to generate spike genes with single or full mutations of B.1.1.529. In brief, 5'-phosphorylated oligos with designed mutations were annealed to the reverse strand of the WT spike gene construct and extended by DNA polymerase. Extension products (forward-stranded fragments) were then ligated together by Taq DNA ligase and subsequently amplified by PCR to generate variants of interest. To verify the sequences of variants, next-generation sequencing libraries were prepared according to a low-volume Nextera sequencing protocol ${ }^{43}$ and sequenced on the Illumina Miseq platform (single-end mode with 50 bp R1). Raw reads were processed using Cutadapt v. $2.1^{44}$ with the default settings to remove adapters, and were then aligned to reference variants sequences using Bowtie2 v.2.3.4 ${ }^{45}$ with the default settings. The resulting reads alignments were then visualized in the Integrative Genomics Viewer ${ }^{46}$ and manually inspected to verify the fidelity of variants. The sequences of the oligos used in variants generation are provided in Extended Data Table 3.

\section{Pseudovirus production}

Pseudoviruses were produced in the vesicular stomatitis virus (VSV) background, in which the native glycoprotein was replaced by that of SARS-CoV-2 and its variants, as previously described ${ }^{24}$. In brief, HEK293T cells were transfected with a spike expression construct with polyethylenimine (PEI) $\left(1 \mathrm{mg} \mathrm{ml}^{-1}\right)$ and cultured overnight at $37^{\circ} \mathrm{C}$ under $5 \% \mathrm{CO}_{2}$, and then infected with VSV-G pseudotyped $\Delta \mathrm{G}$-luciferase $\left(\mathrm{G}^{*} \Delta \mathrm{G}\right.$-luciferase, Kerafast) 1 day after transfection. After $2 \mathrm{~h}$ of infection, cells were washed three times, changed to fresh medium and then cultured for approximately another $24 \mathrm{~h}$ before the supernatants were collected, centrifuged and aliquoted to use in assays.

\section{Pseudovirus neutralization assay}

All viruses were first titrated to normalize the viral input between assays. Heat-inactivated sera or antibodies were first serially diluted in 96 well-plates in triplicate, starting at 1:100 dilution for sera and $10 \mu \mathrm{g} \mathrm{ml}^{-1}$ for antibodies. Viruses were then added and the virus-sample mixture was incubated at $37^{\circ} \mathrm{C}$ for $1 \mathrm{~h}$. Vero-E6 cells (ATCC) were then added at a density of $3 \times 10^{4}$ cells per well and the plates were incubated at $37^{\circ} \mathrm{C}$ for approximately $10 \mathrm{~h}$. Luciferase activity was quantified using the Luciferase Assay System (Promega) according to the manufacturer's instructions using SoftMax Pro v.7.0.2 (Molecular Devices). Neutralization curves and $\mathrm{IC}_{50}$ values were derived by fitting a nonlinear five-parameter dose-response curve to the data in GraphPad Prism v.9.2.

\section{Authentic virus isolation and propagation}

Authentic B.1.1.529 was isolated from a specimen from the respiratory tract of a patient with COVID-19 in Hong Kong by K.-Y. Yuen and colleagues at the Department of Microbiology, The University of Hong Kong. Isolation of WT SARS-CoV-2 was previously described ${ }^{47}$. Viruses were propagated in Vero-E6-TMPRSS2 cells and the sequence was confirmed by next-generation sequencing before use.

\section{Authentic virus neutralization assay}

To measure neutralization of authentic SARS-CoV-2 viruses, Vero-E6-TMPRSS2 cells were first seeded in 96-well plates in cell culture medium (Dulbecco's Modified Eagle Medium (DMEM) $+10 \%$ fetal bovine serum (FBS) $+1 \%$ penicillin-streptomycin) overnight at $37^{\circ} \mathrm{C}$ under $5 \% \mathrm{CO}_{2}$ to establish a monolayer. The next day, sera or antibodies were serially diluted in 96-well plates in triplicate in DMEM + $2 \%$ FBS and then incubated with 0.01 multiplicity of infection of WT SARS-CoV-2 or B.1.1.529 at $37^{\circ} \mathrm{C}$ for $1 \mathrm{~h}$. Sera were diluted from 1:100 dilution and antibodies were diluted from $10 \mu \mathrm{g} \mathrm{ml}^{-1}$. Next, the mixture was overlaid onto cells and further incubated at $37^{\circ} \mathrm{C}$ under $5 \% \mathrm{CO}_{2}$ for approximately $72 \mathrm{~h}$. Cytopathic effects were then scored by plaque assay in a blinded manner. Neutralization curves and $\mathrm{IC}_{50}$ values were derived by fitting a nonlinear five-parameter dose-response curve to the data in GraphPad Prism v.9.2.

\section{Antibody footprint analysis and RBD mutagenesis analysis}

The SARS-CoV-2 spike structure used for displaying epitope footprints and mutations within emerging strains was downloaded from the Protein Data Bank (PDB: 6ZGE). The structures of antibody-spike complexes were also obtained from PDB (7L5B (2-15), 6XDG (REGN10933 and REGN10987), 7L2E (4-18), 7RW2 (5-7), 7C01 (CB6), 7KMG (LY-COV555), 7CDI (Brii-196), 7KS9 (910-30), 7LD1 (DH1047), 7RAL (S2X259), 7LSS (2-7) and 6WPT (S309)). Interface residues were identified using PISA ${ }^{48}$ using the default parameters. The footprint for each antibody was defined by the boundaries of all epitope residues. The border for each footprint was then optimized by ImageMagick v.7.0.10-31 (https:// imagemagick.org). PyMOL v.2.3.2 was used to perform mutagenesis and to generate structural plots (Schrödinger). 
Reporting summary

Further information on research design is available in the Nature Research Reporting Summary linked to this paper.

Data availability

Materials used in this study will be made available under an appropriate Materials Transfer Agreement. All the data are provided in the paper. The structures used for analysis in this study are available from PDB under accession numbers 6ZGE, 7L5B, 6XDG, 7L2E, 7RW2, 7C01,7KMG, 7CDI, 7KS9, 7LD1, 7RAL, 7LSS and 6WPT.

43. Baym, M. et al. Inexpensive multiplexed library preparation for megabase-sized genomes. PLOS ONE 10, e0128036 (2015).

44. Martin, M. Cutadapt removes adapter sequences from high-throughput sequencing reads. EMBnet J. 17, 10-12 (2011).

45. Langmead, B. \& Salzburg, S. L. Fast gapped-read alignment with Bowie 2. Nat. Methods 9, 357-359 (2012).

46. Robinson, J. T. et al. Integrative genomics viewer. Nat. Biotechnol. 29, 24-26 (2011).

47. Thu, H. et al. Comparative tropism, replication kinetics, and cell damage profiling of SARSCoV-2 and SARS-CoV with implications for clinical manifestations, transmissibility, and laboratory studies of COVID-19: an observational study. Lancet Microbe 1, e14-e23 (2020).

48. Krissinel, E. \& Herrick, K. Inference of macromolecular assemblies from crystalline state. J. Mol. Biol. 372, 774-797 (2007).
Acknowledgements We thank the staff at Regeneron Pharmaceuticals, B. Zhang and P. Kwong (NIAID), and B. Dekosky (MIT) for antibodies. This study was supported by funding from the Gates Foundation, the JPB Foundation, A. and P. Cherng, S. Yin, C. Ludwig, D. and R. Wu, Health@InnoHK and the National Science Foundation (MCB-2032259).

Author contributions D.D.H. conceived this project. Lihong Lieu, S.I. and M.W. conducted pseudovirus neutralization experiments. J.F.-W.C., H. Chu, K.K.-H.C., T.T.-T.Y., C.Y., K.K.-W.T. and H. Chen conducted authentic virus neutralization experiments. Y.G. and Z.S. conducted bioinformatic analyses. Liyuan Li and Timing Huang constructed the spike expression plasmids. Y.L. managed the project. J.Y. expressed and purified antibodies. M.T.Y. and M.E.S. provided clinical samples. M.S.N. and Yaoxing Huang contributed to discussions. H.H.W., K.-Y.Y. and D.D.H. directed and supervised the project. Lihong Li, S.I. and D.D.H. analysed the results and wrote the manuscript.

Competing interests Lihong Li, S.I., M.S.N., J.Y., Yaoxing Huang and D.D.H. are inventors on patent applications (WO2021236998) or provisional patent applications $(63 / 271,627)$ filed by Columbia University for a number of SARS-CoV-2 neutralizing antibodies described in this manuscript. Both sets of applications are under review. D.D.H. is a co-founder of MaiMed Biologics and RenBio, consultant to WuXi Biologics and Brii Biosciences, and board director for Vicarious Surgical.

Additional information Supplementary information The online version contains supplementary material available at https://doi.org/10.1038/s41586-021-04388-0.

Correspondence and requests for materials should be addressed to David D. Ho. Peer review information Nature thanks the anonymous reviewers for their contribution to the peer review of this work.

Reprints and permissions information is available at http://www.nature.com/reprints. 


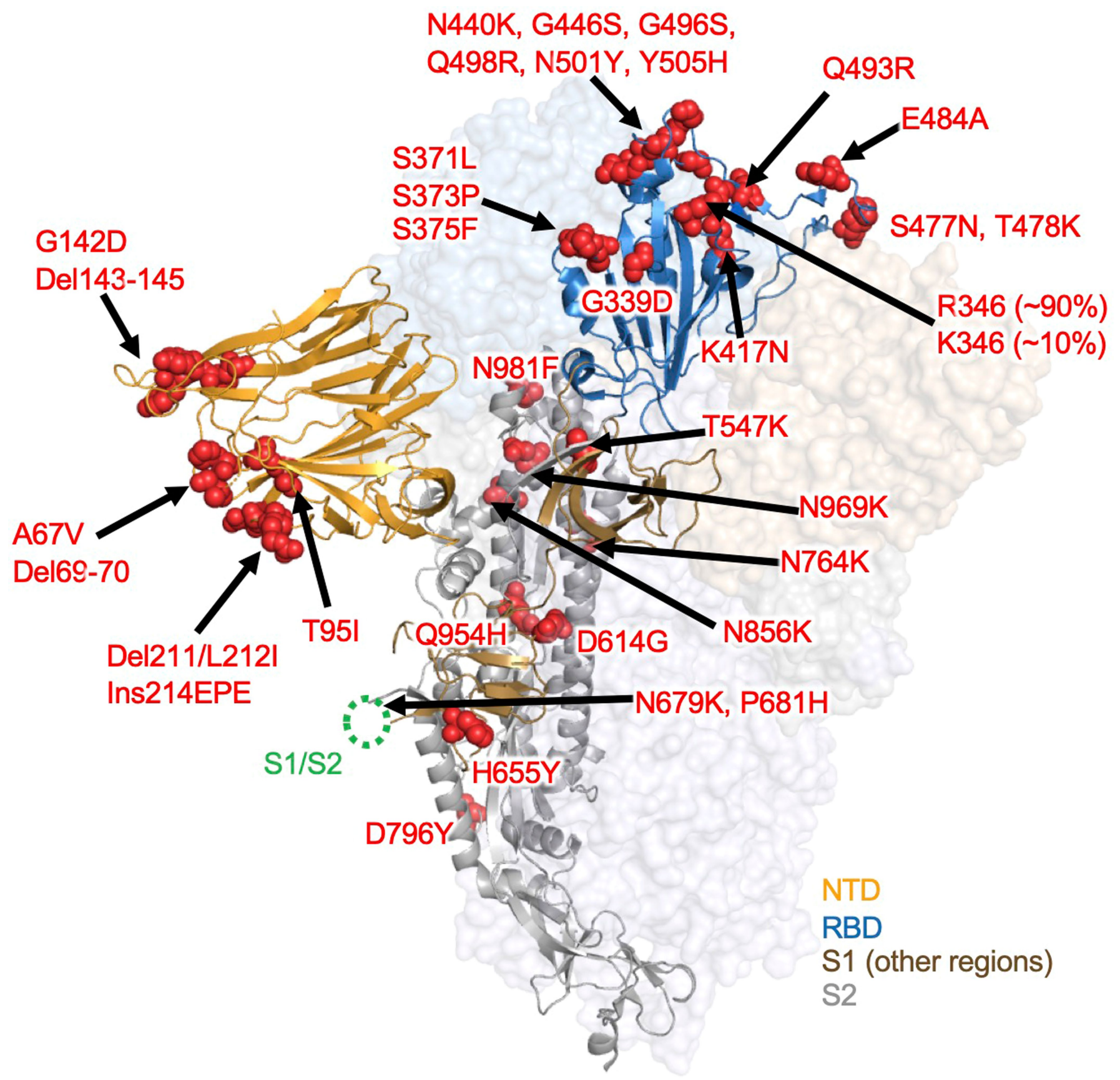

Extended Data Fig.1 | Mutations within B.1.1.529 denoted on the full SARS-CoV-2 spike trimer. The SARS-CoV-2 spike structure was downloaded from PDB 6ZGE. 
a

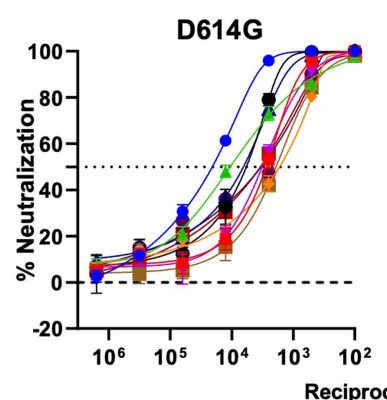

Reciprocal serum dilution

C
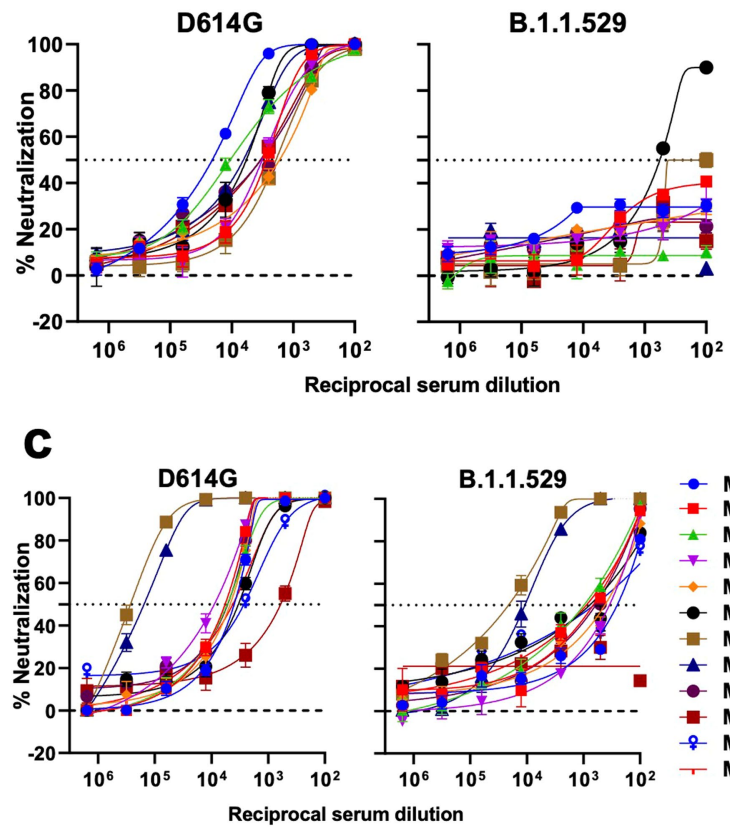

- C1

- $\mathrm{C} 2$

$-\mathrm{C3}$

$\rightarrow \mathrm{C} 4$

- C5

- $\mathrm{C6}$

- $\mathrm{C7}$

- $\mathrm{C8}$

- $\mathrm{C9}$

- C10

e

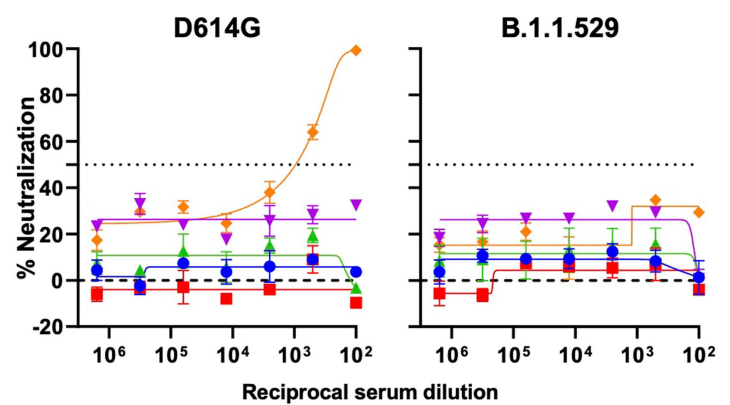

- AZ-1

- AZ-2

- AZ-3

- AZ-4

- AZ-5

Extended Data Fig. 2 | Individual neutralization curves for pseudovirus neutralization assays by serum. Neutralization by a, convalescent sera.

b, Pfizer (BNT162b2) vaccinee sera. c, Moderna (mRNA-1273) vaccinee sera. d, J\&J (Ad26.COV2.S) vaccinee sera.e, AstraZeneca (ChAdOx1 nCoV-19) b

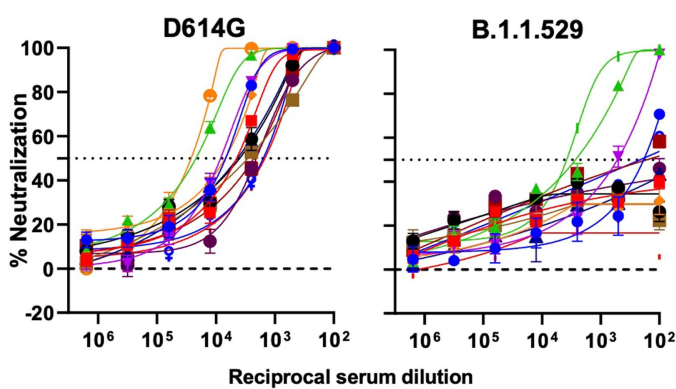

- Pfizer 1

- Pfizer 2

- Pfizer 3

7 Pfizer 4

- Pfizer 5

- Pfizer 6

- Pfizer 7

- Pfizer 8

- Pfizer 9

- Pfizer 10

ㅇ- Pfizer 11

$\begin{array}{ll}\text { Reciprocal serum dilution } & + \text { Pfizer } 13\end{array}$

0

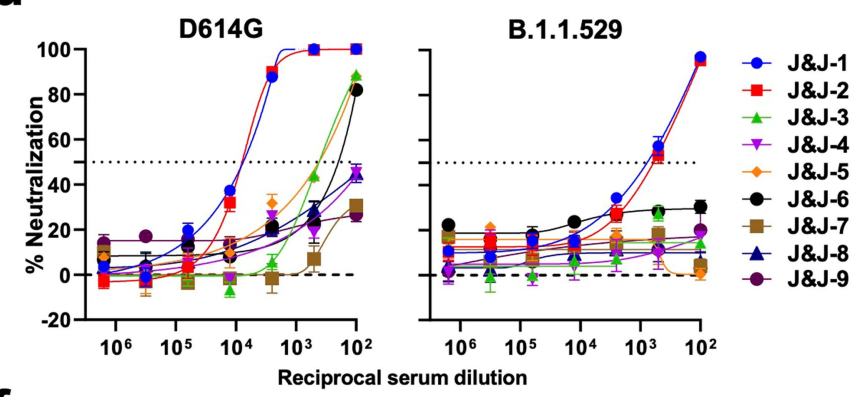

f

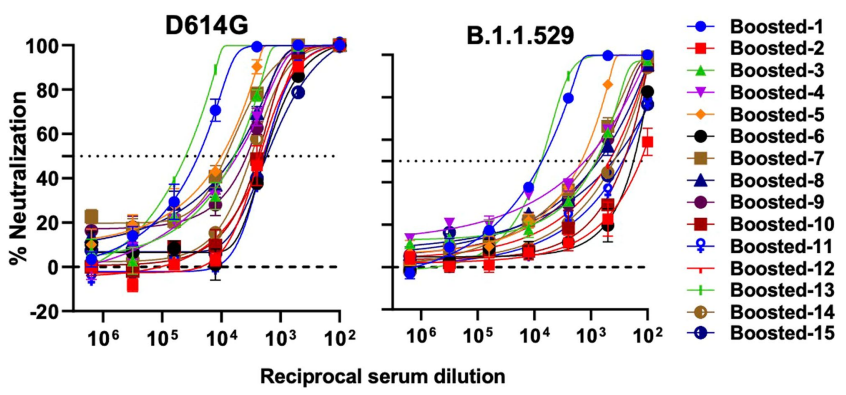

vaccinee sera.f, boosted (three homologous BNT162b2 or mRNA-1273 vaccinations) vaccinee sera. Error bars denote mean \pm standard error of the mean (SEM) for three technical replicates. 

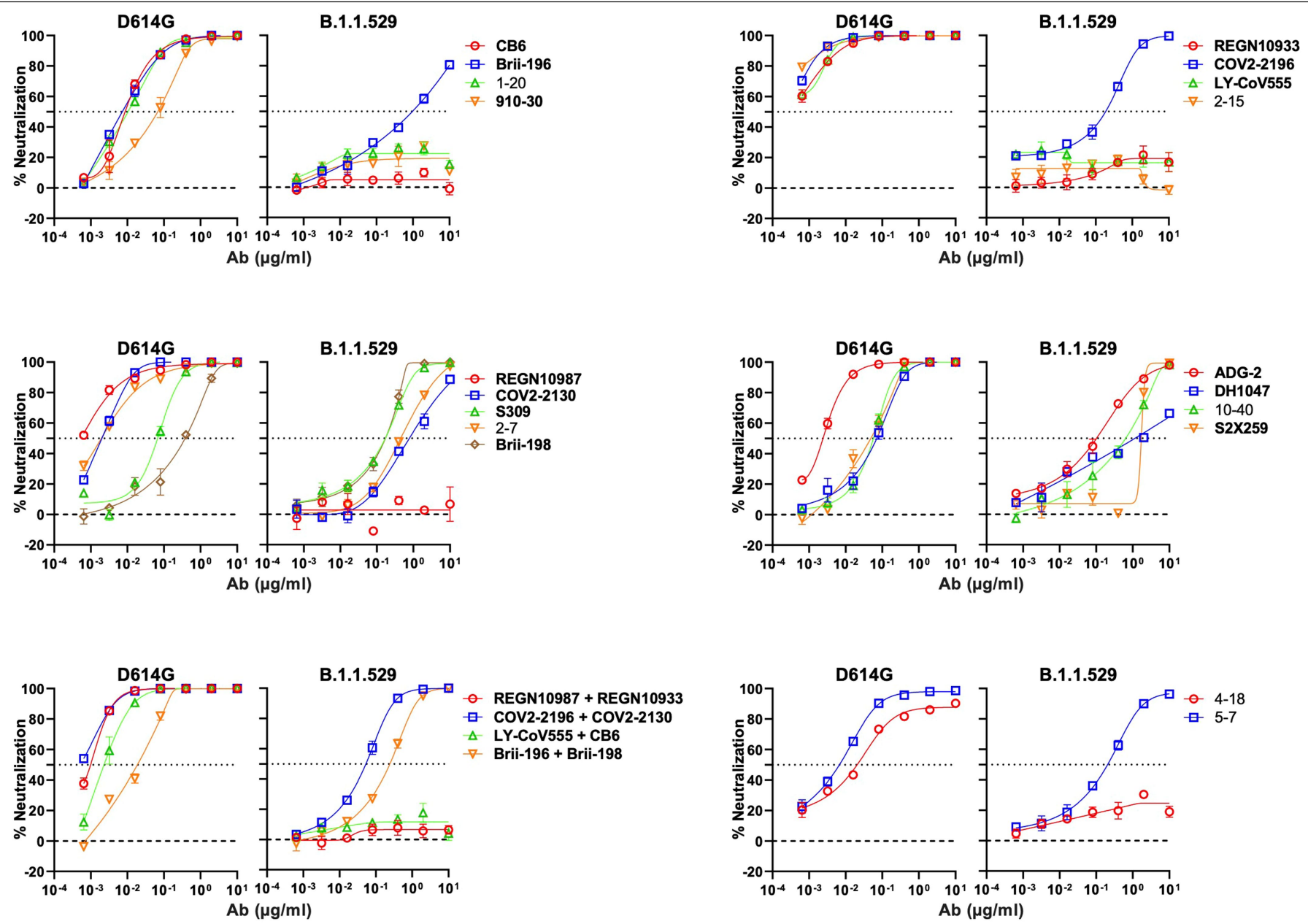

Extended Data Fig. 3 | Individual neutralization curves for pseudovirus neutralization assays by monoclonal antibodies. Error bars denote mean \pm standard error of the mean (SEM) for three technical replicates. 

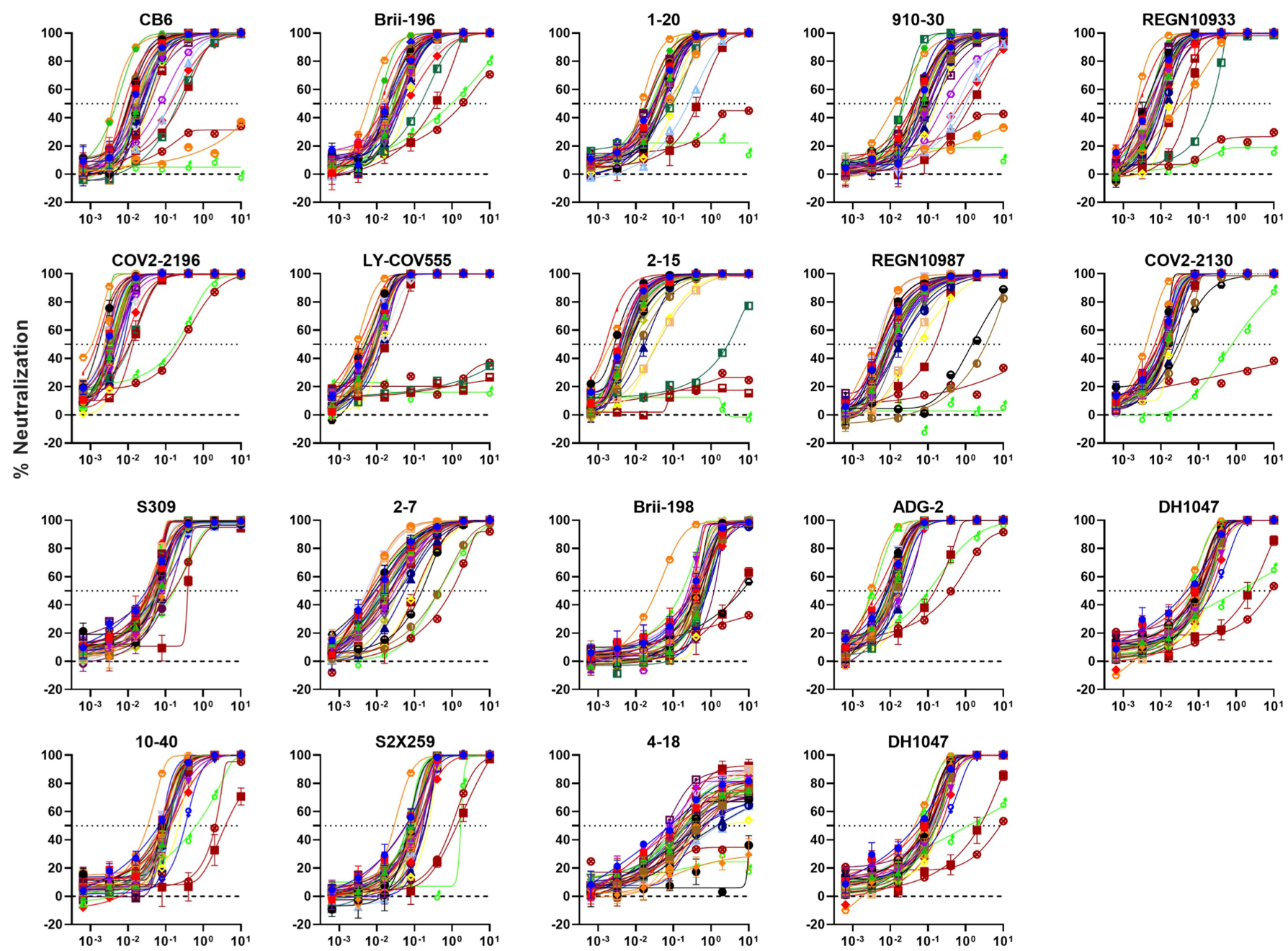

$\mathrm{Ab}(\mu \mathrm{g} / \mathrm{ml})$

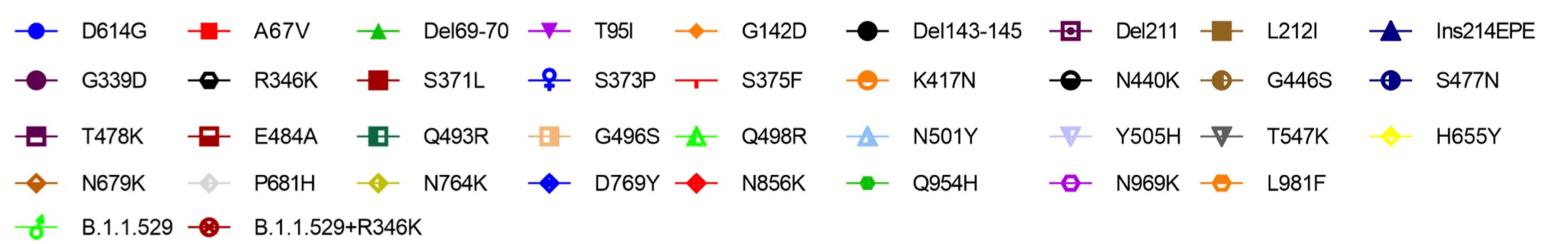

Extended Data Fig. 4 | Individual neutralization curves for pseudovirus neutralization assays by monoclonal antibodies against individual SARS-CoV-2 mutations. Error bars denote mean \pm standard error of the mean (SEM) for three technical replicates. 


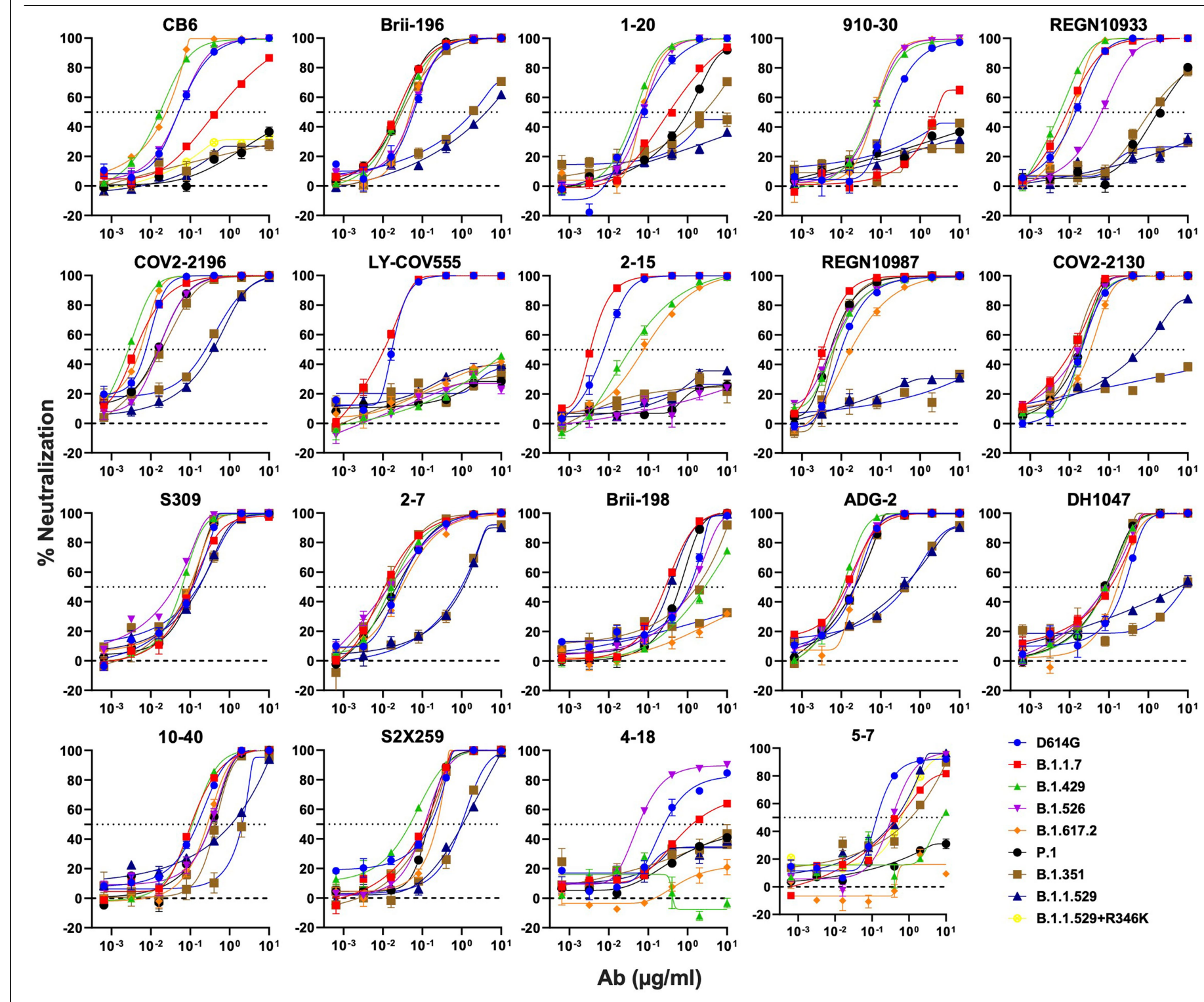

Extended Data Fig. 5 | Individual neutralization curves for pseudovirus neutralization assays by monoclonal antibodies against SARS-CoV-2 variants. Error bars denote mean \pm standard error of the mean (SEM) for three technical replicates. 
Extended Data Table 1 | Demographics and vaccination information for serum samples from convalescent patients used in this study

\begin{tabular}{|cccc|}
\hline Convalescent Sample & Days post-symptoms & Age & Gender \\
& & & \\
C1 & 18 & 57 & Female \\
C2 & 25 & 51 & Male \\
C3 & 29 & 71 & Female \\
C4 & 32 & 50 & Male \\
C5 & 35 & 59 & Male \\
C6 & 120 & 56 & Male \\
C7 & 105 & 54 & Female \\
C8 & 77 & 51 & Female \\
C9 & 18 & 79 & Male \\
C10 & 9 & 45 & Male \\
\hline
\end{tabular}


Extended Data Table 2 | Demographics and vaccination information for serum samples from vaccinated individuals used in this study

\begin{tabular}{|c|c|c|c|c|c|}
\hline Vaccine Sample & Vaccine type & $\begin{array}{c}\text { Days post-vaccination (after } \\
\text { last dose) }\end{array}$ & $\begin{array}{l}\text { Documented COVID } \\
\text { Infection }\end{array}$ & Age & Gender \\
\hline Moderna vaccinee \#1 & mRNA-1273 & 31 & No & 72 & Male \\
\hline Moderna vaccinee \#2 & mRNA-1273 & 19 & No & 38 & Female \\
\hline Moderna vaccinee \#3 & mRNA-1273 & 6 & No & 42 & Male \\
\hline Moderna vaccinee \#4 & mRNA-1273 & 81 & No & 40 & Female \\
\hline Moderna vaccinee \#5 & mRNA-1273 & 123 & No & 40 & Female \\
\hline Moderna vaccinee \#6 & mRNA-1273 & 177 & No & 40 & Female \\
\hline Moderna vaccinee \#7 & mRNA-1273 & 29 & No & 57 & Female \\
\hline Moderna vaccinee \#8 & mRNA-1273 & 74 & No & 57 & Female \\
\hline Moderna vaccinee \#9 & mRNA-1273 & 32 & No & 66 & Female \\
\hline Moderna vaccinee \#10 & mRNA-1273 & 72 & No & 63 & Male \\
\hline Moderna vaccinee \#11 & mRNA-1273 & 74 & No & 68 & Female \\
\hline Moderna vaccinee \#12 & mRNA-1273 & 58 & No & 46 & Female \\
\hline Pfizer vaccinee \#1 & BNT162b2 & 21 & No & 62 & Male \\
\hline Pfizer vaccinee \#2 & BNT162b2 & 36 & No & 62 & Male \\
\hline Pfizer vaccinee \#3 & BNT162b2 & 26 & No & 38 & Male \\
\hline Pfizer vaccinee \#4 & BNT162b2 & 66 & No & 38 & Male \\
\hline Pfizer vaccinee \#5 & BNT162b2 & 22 & No & 57 & Female \\
\hline Pfizer vaccinee \#6 & BNT162b2 & 61 & No & 57 & Female \\
\hline Pfizer vaccinee \#7 & BNT162b2 & 20 & No & 55 & Male \\
\hline Pfizer vaccinee \#8 & BNT162b2 & 16 & No & 64 & Female \\
\hline Pfizer vaccinee \#9 & BNT162b2 & 32 & No & 68 & Male \\
\hline Pfizer vaccinee \#10 & BNT162b2 & 20 & No & 35 & Male \\
\hline Pfizer vaccinee \#11 & BNT162b2 & 15 & No & 48 & Female \\
\hline Pfizer vaccinee \#12 & BNT162b2 & 21 & No & 45 & Male \\
\hline Pfizer vaccinee \#13 & BNT162b2 & 213 & Yes & 66 & Male \\
\hline J\&J vaccinee \#1 (BEI Cat. \#NRH-10818) & Ad26.COV2.S & 55 & Yes & 50 & Female \\
\hline J\&J vaccinee \#2 (BEI Cat. \#NRH-10819) & Ad26.COV2.S & 61 & Yes & 50 & Female \\
\hline J\&J vaccinee \#3 (BEI Cat. \#NRH-10835) & Ad26.COV2.S & 186 & Unknown & 43 & Female \\
\hline J\&J vaccinee \#4 (BEI Cat. \#NRH-10845) & Ad26.COV2.S & 69 & Unknown & 28 & Female \\
\hline J\&J vaccinee \#5 (BEI Cat. \#NRH-10823) & Ad26.COV2.S & 50 & No & 42 & Female \\
\hline J\&J vaccinee \#6 (BEI Cat. \#NRH-10834) & Ad26.COV2.S & 175 & Unknown & 43 & Female \\
\hline J\&J vaccinee \#7 (BEI Cat. \#NRH-10839) & Ad26.COV2.S & 39 & No & 47 & Male \\
\hline J\&J vaccinee \#8 (BEI Cat. \#NRH-10844) & Ad26.COV2.S & 60 & Unknown & 28 & Female \\
\hline J\&J vaccinee \#9 (BEI Cat. \#NRH-10824) & Ad26.COV2.S & 51 & No & 43 & Male \\
\hline AZ vaccinee \#1 (BEI Cat. \#NRH-10817) & ChAdOx1 nCoV-19 & 158 & Unknown & 73 & Male \\
\hline AZ vaccinee \#2 (BEI Cat. \#NRH-10814) & ChAdOx1 nCoV-19 & 152 & Unknown & 36 & Female \\
\hline AZ vaccinee \#3 (BEI Cat. \#NRH-10815) & ChAdOx1 nCoV-19 & 159 & Unknown & 36 & Female \\
\hline AZ vaccinee \#4 (BEI Cat. \#NRH-10811) & ChAdOx1 nCoV-19 & 142 & Yes & 26 & Female \\
\hline AZ vaccinee \#5 (BEI Cat. \#NRH-3083) & ChAdOx1 nCoV-19 & 91 & Unknown & 56 & Female \\
\hline Boosted sera \#1 & mRNA-1273/mRNA-1273 & 28 & No & 66 & Female \\
\hline Boosted sera \#2 & BNT162b2/BNT162b2 & 30 & No & 68 & Male \\
\hline Boosted sera \#3 & BNT162b2/BNT162b2 & 14 & No & 64 & Female \\
\hline Boosted sera \#4 & BNT162b2/BNT162b2 & 34 & No & 55 & Male \\
\hline Boosted sera \#5 & BNT162b2/BNT162b2 & 34 & No & 45 & Male \\
\hline Boosted sera \#6 & BNT162b2/BNT162b2 & 15 & No & 50 & Female \\
\hline Boosted sera \#7 & BNT162b2/BNT162b2 & 15 & No & 48 & Female \\
\hline Boosted sera \#8 & BNT162b2/BNT162b2 & 29 & No & 71 & Male \\
\hline Boosted sera \#9 & BNT162b2/BNT162b2 & 90 & No & 59 & Male \\
\hline Boosted sera \#10 & BNT162b2/BNT162b2 & 33 & No & 45 & Male \\
\hline Boosted sera \#11 & BNT162b2/BNT162b2 & 87 & No & 66 & Female \\
\hline Boosted sera \#12 & BNT162b2/BNT162b2 & 84 & No & 26 & Male \\
\hline Boosted sera \#13 & mRNA-1273/mRNA-1273 & 23 & No & 28 & Female \\
\hline Boosted sera \#14 & BNT162b2/BNT162b2 & 14 & No & 78 & Male \\
\hline Boosted sera \#15 & BNT162b2/BNT162b2 & 14 & No & 75 & Female \\
\hline
\end{tabular}


Extended Data Table 3 | Oligos used to construct spike expression plasmids

\begin{tabular}{|c|c|c|}
\hline Oligo name & Targeted mutations & Oligo sequence \\
\hline O_single_mutant1 & A67V & ATGTGACCTGGTTCCATGTGATCCATGTGTCTGGCACCAATGGCACC \\
\hline O_single_mutant2 & Del69-70 & CTGGTTCCATGCCATCTCTGGCACCAATGGCAC \\
\hline O_single_mutant3 & T95I & CTTTGCCAGCATCGAGAAGAGCAACATCATC \\
\hline O_single_mutant4 & Del143-145 & TGTAATGACCCATTCCTGGGACACAAGAACAACAAGTCCTGGATG \\
\hline O_single_mutant5 & G142D & GTAATGACCCATTCCTGGACGTCTACTACCACAAG \\
\hline O_single_mutant6 & Del211 & ACACACACCAATCCTGGTGAGGGACCTG \\
\hline O_single_mutant7 & L212I & CACACCAATCAACATCGTGAGGGACCTGCC \\
\hline O_single_mutant8 & Ins214EPE & ACCAATCAACCTGGTGAGGGAGCCCGAGGACCTGCCACAGGGCTT \\
\hline O_single_mutant9 & G339D & CTGTGTCCATTTGACGAGGTGTTCAATGCCAC \\
\hline O_single_mutant10 & R346K & TGTTCAATGCCACCAAGTTTGCCTCTGTCTATGCCTG \\
\hline O_single_mutant11 & S371F & CTCTGTGCTCTACAACTTTGCCTCCTTCAGCAC \\
\hline O_single_mutant12 & S371L & CTCTGTGCTCTACAACCTGGCCTCCTTCAGCAC \\
\hline O_single_mutant13 & S373P & СTCTACAACTCTGCССССTTCAGCACCTTCAAG \\
\hline O_single_mutant14 & S375F & CAACTCTGCCTCCTTCTTCACCTTCAAGTGTTATGG \\
\hline O_single_mutant15 & $\mathrm{K} 417 \mathrm{~N}$ & CCCCTGGACAAACAGGCAACATTGCTGACTACAACTACAAACTGC \\
\hline O_single_mutant16 & N440K & CCTGGAACAGCAACAAGCTGGACAGCAAGGTG \\
\hline O_single_mutant17 & G446S & GGACAGCAAGGTGAGCGGCAACTACAACTAC \\
\hline O_single_mutant18 & S477N & GATTTACCAGGCTGGCAACACACCATGTAATG \\
\hline O_single_mutant19 & T478K & CAGGCTGGCAGCAAGCCATGTAATGGAGTGGA \\
\hline O_single_mutant20 & E484A & GTAATGGAGTGGCCGGCTTCAACTGTTAC \\
\hline O_single_mutant21 & Q493R & GTTACTTTCCACTCAGATCCTATGGCTTCCAAC \\
\hline O_single_mutant22 & G496S & CACTCCAATCCTATAGCTTCCAACCAACCAATG \\
\hline O_single_mutant23 & Q498R & CAATCCTATGGCTTCAGACCAACCAATGGAGTGGG \\
\hline O_single_mutant24 & N501Y & CTTCCAACCAACCTACGGAGTGGGCTACCAACC \\
\hline O_single_mutant25 & Y505H & AATGGAGTGGGCCACCAACCATACAGG \\
\hline O_single_mutant26 & T547K & CTTCAATGGACTGAAGGGCACAGGAGTGCTGAC \\
\hline O_single_mutant27 & H655Y & CTGATTGGAGCAGAGTACGTGAACAACTCCTATG \\
\hline O_single_mutant28 & N679K & CCAGACCCAGACCAAGAGCCCAAGGAGGGCA \\
\hline O_single_mutant29 & $\mathrm{P} 681 \mathrm{H}$ & CCCAGACCAACAGCAGAAGGAGGGCAAGGTCTGTGGC \\
\hline O_single_mutant30 & N764K & GTACCCAACTTAAGAGGGCTCTGACAGGC \\
\hline O_single_mutant31 & D769Y & GACACCTCCAATCAAGTACTTTGGAGGCTTC \\
\hline O_single_mutant32 & N856K & GTGCCCAGAAGTTCAAGGGACTGACAGTGCTG \\
\hline O_single_mutant33 & Q954H & CAAGATGTGGTGAACCACAATGCCCAGGCTCTG \\
\hline O_single_mutant34 & N969K & GCAACTTTCCAGCAAGTTTGGAGCCATCTCCTC \\
\hline O_single_mutant35 & L981F & GTGCTGAATGACATCTTCAGCAGACTGGACAAGGTGGAGG \\
\hline O_multiple_oligo1 & A67V, Del69-70 & TGGTTCCATGTGATCTCTGGCACCAATGG \\
\hline O_multiple_oligo2 & T95I & CTTTGCCAGCATCGAGAAGAGCAAC \\
\hline O_multiple_oligo3 & G142D, Del143-145 & GACCCATTCCTGGACCACAAGAACAACAAGTC \\
\hline O_multiple_oligo4 & L212I, Ins214EPE & CACACACCAATCATCGTGAGGGAGCCCGAGGACCTGCCACAGGGCTTC \\
\hline O_multiple_oligo5 & G339D & TGTGTCCATTTGACGAGGTGTTCAATG \\
\hline O_multiple_oligo6 & S371L, S373P, S375F & TGTGCTCTACAACCTGGCCCCCTTCTTCACCTTCAAGTGTTATG \\
\hline O_multiple_oligo7 & $\mathrm{K} 417 \mathrm{~N}$ & GGACAAACAGGCAACATTGCTGACTACA \\
\hline O_multiple_oligo8 & N440K, G446S & GCAACAAGCTGGACAGCAAGGTGAGCGGCAACTACAA \\
\hline O_multiple_oligo9 & S477N, T478K, E484A & ACCAGGCTGGCAACAAGCCATGTAATGGAGTGGCCGGCTTCAACTGT \\
\hline O_multiple_oligo10 & $\begin{array}{l}\text { Q493R, G496S, Q498R, } \\
\text { N501Y, Y505H }\end{array}$ & $\begin{array}{l}\text { TACTTTCCACTCAGATCCTATAGCTTCAGACCAACCTACGGAGTGGGCCACCAACCATACAGG } \\
\text { GTGGTGGTGCTGTCCTTTGA }\end{array}$ \\
\hline O_multiple_oligo11 & T547K & GGACTGAAGGGCACAGGAG \\
\hline O_multiple_oligo12 & D614G & CTCTACCAGGGCGTGAACTGTAC \\
\hline O_multiple_oligo13 & H655Y & TTGGAGCAGAGTACGTGAACAACTC \\
\hline O_multiple_oligo14 & N679K, P681H & CAGACCAAGAGCCACAGGAGGGCAAGG \\
\hline O_multiple_oligo15 & N764K & CCAACTTAAGAGGGCTCTGACAG \\
\hline O_multiple_oligo16 & D796Y & CCTCCAATCAAGTACTTTGGAGGCTTC \\
\hline O_multiple_oligo17 & N856K & CAGAAGTTCAAGGGACTGACAGTGCTG \\
\hline O_multiple_oligo18 & Q954H & GTGGTGAACCACAATGCCCAGGCTC \\
\hline O_multiple_oligo19 & N969K & AACTTTCCAGCAAGTTTGGAGCCATCTCCTC \\
\hline O_multiple_oligo20 & L981F & AATGACATCTTCAGCAGACTGGACAAGGTGGAGGCTGAGGTCCAGATTG \\
\hline
\end{tabular}




\section{nature portfolio}

Corresponding author(s): David D. Ho

Last updated by author(s): Dec 20, 2021

\section{Reporting Summary}

Nature Portfolio wishes to improve the reproducibility of the work that we publish. This form provides structure for consistency and transparency

in reporting. For further information on Nature Portfolio policies, see our Editorial Policies and the Editorial Policy Checklist.

\section{Statistics}

For all statistical analyses, confirm that the following items are present in the figure legend, table legend, main text, or Methods section.

n/a Confirmed

$\bigotimes$ The exact sample size $(n)$ for each experimental group/condition, given as a discrete number and unit of measurement

$\square$ \A statement on whether measurements were taken from distinct samples or whether the same sample was measured repeatedly

The statistical test(s) used AND whether they are one- or two-sided

$\square$ Only common tests should be described solely by name; describe more complex techniques in the Methods section.

\ $\square$ A description of all covariates tested

Х $\square$ A description of any assumptions or corrections, such as tests of normality and adjustment for multiple comparisons

$\checkmark$ A full description of the statistical parameters including central tendency (e.g. means) or other basic estimates (e.g. regression coefficient)

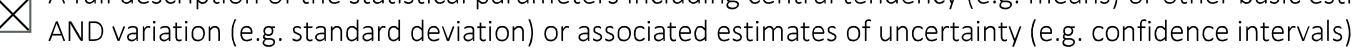

For null hypothesis testing, the test statistic (e.g. $F, t, r$ ) with confidence intervals, effect sizes, degrees of freedom and $P$ value noted

Give $P$ values as exact values whenever suitable.

Х $\square$ For Bayesian analysis, information on the choice of priors and Markov chain Monte Carlo settings

Х $\square$ For hierarchical and complex designs, identification of the appropriate level for tests and full reporting of outcomes

Х $\square$ Estimates of effect sizes (e.g. Cohen's $d$, Pearson's $r$ ), indicating how they were calculated

Our web collection on statistics for biologists contains articles on many of the points above.

\section{Software and code}

Policy information about availability of computer code

Data collection SoftMax Pro 7.0.2 (Molecular Devices, LLC) was used to measure luminescence in the pseudovirus neutralization assays.

Data analysis GraphPad Prism (version 9.2) was used for data visualization and for statistical tests. Cutadapt (version 2.1) was used for processing of raw reads from next-generation sequencing. Bowtie2 (version 2.3.4) was used for alignment of reads to sequences. PISA was used for identifying antibody-spike interface residues. Antibody footprints were optimized by ImageMagick 7.0.10-31. PyMOL (version 2.3.2) was used for RBD mutagenesis analysis and for visualization.

For manuscripts utilizing custom algorithms or software that are central to the research but not yet described in published literature, software must be made available to editors and reviewers. We strongly encourage code deposition in a community repository (e.g. GitHub). See the Nature Portfolio guidelines for submitting code \& software for further information.

\section{Data}

Policy information about availability of data

All manuscripts must include a data availability statement. This statement should provide the following information, where applicable:

- Accession codes, unique identifiers, or web links for publicly available datasets

- A description of any restrictions on data availability

- For clinical datasets or third party data, please ensure that the statement adheres to our policy

Materials used in this study will be made available under an appropriate Materials Transfer Agreement. All the data are provided in the paper. The structures used for analysis in this study are available from PDB under IDs 6ZGE, 7L5B, 6XDG, 7L2E, 7RW2, 7C01, 7KMG, 7CDI, 7KS9, 7LD1, 7RAL, 7LSS, and 6WPT. 


\section{Field-specific reporting}

Please select the one below that is the best fit for your research. If you are not sure, read the appropriate sections before making your selection.

$\bigotimes$ Life sciences $\quad \square$ Behavioural \& social sciences $\quad \square$ Ecological, evolutionary \& environmental sciences

For a reference copy of the document with all sections, see nature.com/documents/nr-reporting-summary-flat.pdf

\section{Life sciences study design}

All studies must disclose on these points even when the disclosure is negative.

Sample size We used similar sample sizes as in previous work (e.g. Wang et al 2021, Nature), which we had previously determined to be sufficient sample sizes for comparisons between groups for these experiments.

Data exclusions No data were excluded.

Replication The key results, the resistance of R346K, S371L, B.1.1.529, and B.1.1.529+R346K to monoclonal antibodies in pseudoviruses, and serum neutralization of authentic viruses, were repeated twice independently in technical triplicate with similar results. The results that are shown are representative. Other experiments were conducted in technical triplicate and not repeated.

Randomization As this is an observational study, randomization is not relevant.

Blinding As this is an observational study, investigators were not blinded.

\section{Reporting for specific materials, systems and methods}

We require information from authors about some types of materials, experimental systems and methods used in many studies. Here, indicate whether each material, system or method listed is relevant to your study. If you are not sure if a list item applies to your research, read the appropriate section before selecting a response.

Materials \& experimental systems

\begin{tabular}{l|l}
\multicolumn{2}{l}{ Methods } \\
\hline n/a & Involved in the study \\
$\bigotimes$ & $\square$ ChIP-seq \\
$\searrow$ & $\square$ Flow cytometry \\
$\searrow$ & $\square$ MRI-based neuroimaging
\end{tabular}

$\mathrm{n} / \mathrm{a}$ Involved in the study

$\square$ Antibodies

$\square$ Eukaryotic cell lines

Х $\square$ Palaeontology and archaeology

Х $\square$ Animals and other organisms

$\square \bigotimes$ Human research participants

X $\square$ Clinical data

Х Dual use research of concern

\section{Antibodies}

Antibodies used

All of the antibodies used in this study were produced in our laboratory or received from other laboratories. 1-20, 2-15, 5309, 2-7 ADG-2, DH1047, 10-40, S2X259, 4-18, and 5-7 were expressed and purified in-house as described previously in Liu et al 2020, Nature. REGN10987, REGN10933, COV2-2196, and COV2-2130 were provided by Regeneron Pharmaceuticals, Brii-196 and Brii-198 were provided by Brii Biosciences, CB6 was provided by Baoshan Zhang and Peter Kwong (NIH), and 910-30 was provided by Brandon DeKosky (MIT).

Validation

All of the antibodies have been validated in previous studies both by binding to SARS-CoV-2 spike and neutralization of SARS-CoV-2 (both pseudovirus and authentic virus), and when applicable, have been confirmed to give similar results as that described in publications by other groups. Specifically, 1-20 and 4-18 were tested in Liu et al 2020, Nature, CB6, Brii-196, 910-30, REGN10933, COV2-2196, LY-CoV555, 2-15, REGN10987, COV2-2130, S309, 2-7, Brii-198, and 5-7 were tested in Wang et al 2021, Nature, and ADG-2, DH1047, 10-40, and S2X259 were tested in Liu et al 2021, bioRxiv.

\section{Eukaryotic cell lines}

Policy information about cell lines

Cell line source(s)

Expi293 cells were obtained from Thermo Fisher (Catalog \#A14527), Vero E6 cells were obtained from ATCC (Catalog\# CRL-1586), HEK293T cells were obtained from ATCC (Catalog\# CRL-3216), and Vero-E6-TMPRSS2 cells were obtained from JCRB (Catalog\# JCRB1819). 


\section{Mycoplasma contamination}

Commonly misidentified lines (See ICLAC register)

\section{Cell lines tested mycoplasma negative.}

No commonly misidentified cell lines were used in this study.

\section{Human research participants}

Policy information about studies involving human research participants

Population characteristics

Population characteristics are described in detail for each individual in Extended Data Table 1 and 2. Convalescent samples had the following ranges: 9-120 days post-symptoms, 45-79 years old, 4/10 female, 6/10 male. We presume all of these individuals were infected with the wild-type strain of SARS-CoV-2 as these samples were collected in Spring of 2020. Vaccinee samples had the following ranges: 6-213 days post-vaccination, 26-78 years old, 12/54 two mRNA-1273 vaccinations, 13/54 two BNT162b2 vaccinations, 9/54 Ad26.COV2.S vaccination, 5/54 two ChAdOx1 nCoV-19 vaccinations, 2/54 three mRNA-1273 vaccinations, $13 / 54$ three BNT162b2 vaccinations, 4/54 previously infected, $8 / 54$ unknown previous infection status, $42 / 54$ uninfected, $31 / 54$ female, $23 / 54$ male.

Recruitment

For convalescent sera, convalescing patients volunteered and were enrolled in an observational cohort study at Columbia University Irving Medical Center in Spring of 2020. For the BNT162b2 and mRNA-1273 vaccinee sera, individuals volunteered and were enrolled in an observational cohort study at Columbia University Irving Medical Center to study the immunological responses to SARS-CoV-2 in individuals who had received COVID-19 vaccines. Ad26.COV2.S and ChAdOx1 nCoV-19 vaccinee serum samples were received from BEI Resources. Self-selection biases may have affected the demographics of the enrolled population, but are not expected to have impacted the results of this study. High titer samples were specifically chosen within each of the serum groups so that fold-change in titer could be better determined, as also discussed in the manuscript.

Ethics oversight

All collections were conducted under protocols reviewed and approved by the Institutional Review Board of Columbia University. All participants provided written informed consent.

Note that full information on the approval of the study protocol must also be provided in the manuscript. 